\title{
Biomarkers of cereal food intake
}

\author{
Rikard Landberg ${ }^{{ }^{*}}$, Kati Hanhineva ${ }^{2}$, Kieran Tuohy ${ }^{3}$, Mar Garcia-Aloy ${ }^{4,5}$, Izabela Biskup ${ }^{1}$, Rafael Llorach ${ }^{4,5}$, \\ Xiaofei Yin ${ }^{6}$, Lorraine Brennan ${ }^{6}$ and Marjukka Kolehmainen ${ }^{2}$
}

\begin{abstract}
Background/objectives: Cereal foods are major contributors to the daily energy, protein, and dietary fiber intake all over the world. The role of cereals in human health is dependent on whether they are consumed as refined or whole grain and on cereal species. To unravel the underlying mechanisms of health effects attributed to specific cereal foods and to provide more precise dietary advice, there is a need for improved dietary assessment of wholegrain intake. Dietary biomarkers of specific cereals, different fractions or cereal-containing foods could offer such a possibility. The aim of this review was to summarize the current status on biomarkers of different cereals, fractions, and specific cereal foods.

Subjects and methods: A literature review was conducted and putative biomarkers of different cereals and pseudo-cereals (wheat, oats, rye, barley, rice, and quinoa) as well as for different grain fractions (whole grain, refined grain, bran) and foods were summarized and discussed.

Results: Several putative biomarkers have been suggested for different cereals, due to their unique presence in these grains. Among the biomarkers, odd-numbered alkylresorcinols are the most well-studied and -evaluated biomarkers and reflect whole-grain wheat and rye intake. Even-numbered alkylresorcinols have been suggested to reflect quinoa intake. Recent studies have also highlighted the potential of avenanthramides and avenacosides as specific biomarkers of oat intake, and a set of biomarkers have been suggested to reflect rice bran intake. However, there are yet no specific biomarkers of refined grains. Most biomarker candidates remain to be evaluated in controlled interventions and free-living populations before applied as biomarkers of intake in food and health studies.
\end{abstract}

Conclusion: Several putative biomarkers of different cereals have been suggested and should be validated in human studies using recently developed food intake biomarker validation criteria.

Keywords: Cereals, Biomarkers, Whole grain, Alkylresorcinols, Cinnamic acids, Phenolic acids, Benzoxazinoids,

Avenanthramides, Avenacosides

\section{Background}

Cereal foods constitute a major food group, and they are one of the main contributors to energy and dietary fiber intake in the diet all over the world [1]. Today, cereals are mostly consumed as refined grains, i.e., the nutrientrich bran and germ have been removed. However, whole grain-based foods, i.e., where all parts of the grain kernel are present in cracked, intact, or milled form, is reaching wider acceptance among consumers. Whole-grain foods are advocated by governmental authorities in many countries due to beneficial health effects [2]. Whole-

\footnotetext{
* Correspondence: rikard.landberg@chalmers.se

'Department of Biology and Biological Engineering, Division of Food and Nutrition Science, Chalmers University of Technology, SE-412 96 Gothenburg, Sweden

Full list of author information is available at the end of the article
}

grain food intake has been consistently associated with lower risk of non-communicable diseases such as obesity, cardiovascular disease, type 2 diabetes, and colorectal cancer in different populations [3-7], whereas a high intake of refined grains has been associated with no or even adverse health outcomes $[4,8,9]$. Whole grains are rich in dietary fiber, vitamins, minerals, unsaturated fatty acids, and phytochemicals, all of which may contribute to protective effects [10]. Moreover, the native structure of the food raw material as well as process induced structural changes that might encapsulate nutrients, slow digestion, and absorption could also play a role for health especially in the gut [11]. Specific dietary fibers, such as $\beta$-glucans, the fructans, and resistant starches, including process-induced resistant starch commonly

(C) The Author(s). 2019 Open Access This article is distributed under the terms of the Creative Commons Attribution 4.0 International License (http://creativecommons.org/licenses/by/4.0/), which permits unrestricted use, distribution, and 
found in whole grains, could induce gut microbiota fermentation in the large intestine, which has been linked to beneficial health effects $[12,13]$.

While observational studies $[3-5,14,15]$ have provided consistent evidence for a beneficial effect of high wholegrain intake in chronic disease prevention, the outcomes from short- to long-term randomized controlled trials are less consistent $[16,17]$. Yet, randomized controlled trials investigating the role of whole-grain intake for primary prevention of non-communicable disease have not been reported, due to large challenges related to costs of such trials and problems to ensure compliance over long periods of time. Instead, short-term dietary interventions to address effects on established biomarkers or risk markers for non-communicable diseases have been conducted to investigate the role of separate grains and mixed whole grains on cardiometabolic risk factors, but also short- to intermediate-term studies have been shown to have problems with compliance [17-19].

A problem in observational studies is that whole-grain intake is associated with an overall healthy lifestyle and dietary pattern, and it is difficult to study the impact of whole grains per se on health outcomes, despite adjustment for confounding factors [20]. Moreover, and probably more importantly, the dietary instruments typically used to assess whole-grain intake in observational studies lack the precision required to accurately measure the intake of different grains separately. Various cereals differ in the content and composition of constituents thought to exert health effects, but this has typically not been accounted for in observational studies [21-23]. Another challenge for accurate assessment of the habitual whole-grain intake with common self-reporting techniques such as food frequency questionnaires, dietary recalls, or food records is that consumers may have difficulties in distinguishing/identifying different grains and to understand portion sizes, in addition to wellknown effects of under- and over-reporting. Furthermore, whole-grain products have a large variation in whole-grain content, which affects the precision of estimates [24]. Moreover, no uniform definition of whole-grain products or serving size has been used across studies [25-27]. This may lead to misclassification, which is likely to attenuate the association between whole grain and disease towards null and preventing existing associations with disease outcomes to be revealed or cause underestimation of associations that may be stronger than observed [28].

Using dietary biomarkers that reflect the intake of specific whole grains, grain fractions, and refined grains could be a strategy to improve whole-grain intake ranking in observational studies as well as to address compliance in dietary intervention studies [29-32]. Dietary biomarkers may also be combined with traditional methods to improve the accuracy of intake estimations [33]. However, only a few dietary biomarkers that reflect specific wholegrain intakes have been suggested [34] whereas no biomarkers of refined grains have been described.

The aim of the present review is to provide an updated overview of potential biomarkers of different cereals, including different species, whole grains, refined grains as well as specific grain fractions.

\section{Literature search}

The reviewing process conducted made use of all elements of the PRISMA statement [35] that were relevant for a search for literature on cereal biomarkers. In brief, original research papers and reviews were searched in at least two databases, such as CAB Abstracts, Scopus, and ISI Web of Knowledge using combinations of the grouped search terms (biomarker* OR marker* OR metabolite* OR biokinetics OR biotransformation OR metabolism) AND (trial OR experiment OR study OR intervention) AND (human* OR men OR women OR patient* OR volunteer* OR participant) AND (urine OR plasma OR serum OR blood OR excretion) AND (intake OR meal or diet OR ingestion OR consumption OR eating OR drink" OR administration) AND (wheat* OR rye OR oat* OR barley OR rice OR sorghum OR corn OR maize OR germ OR endosperm OR bran OR wholegrain OR whole-grain OR "whole grain" OR bread" OR cereal" OR flour* OR pasta*). The research was limited to papers in the English language, while no restriction was applied for the publication date. The research papers with identification or use of potential biomarkers of cereal intake were selected by one or more skilled researchers from the list of retrieved references in a process outlined in Fig. 1. Additional papers were identified from reference lists in these papers and from reviews or book chapters identified through the search. For each potential biomarker identified an additional search was conducted with ("the name and synonyms of the compound" OR "the name and synonyms of any parent compound") AND (biomarker" OR marker* OR metabolite* OR biokinetics OR biotransformation) in order to identify potential other foods containing the biomarker or its precursor. In this second step, PubMed, Scifinder, and Google Scholar were also used as search platforms, along with the databases listed above. This second search was used to evaluate the apparent specificity of proposed biomarkers. The literature search was conducted in 2016 and papers published until the end of 2016 were included. A complementary search was conducted in a similar way and additional papers published until June 2018 were added to the literature list.

\section{Classification of cereal biomarkers}

Dietary biomarkers may reflect intake or efficacy, depending on whether the biomarker is a compound resulting from the consumed dietary item, or if it is an 


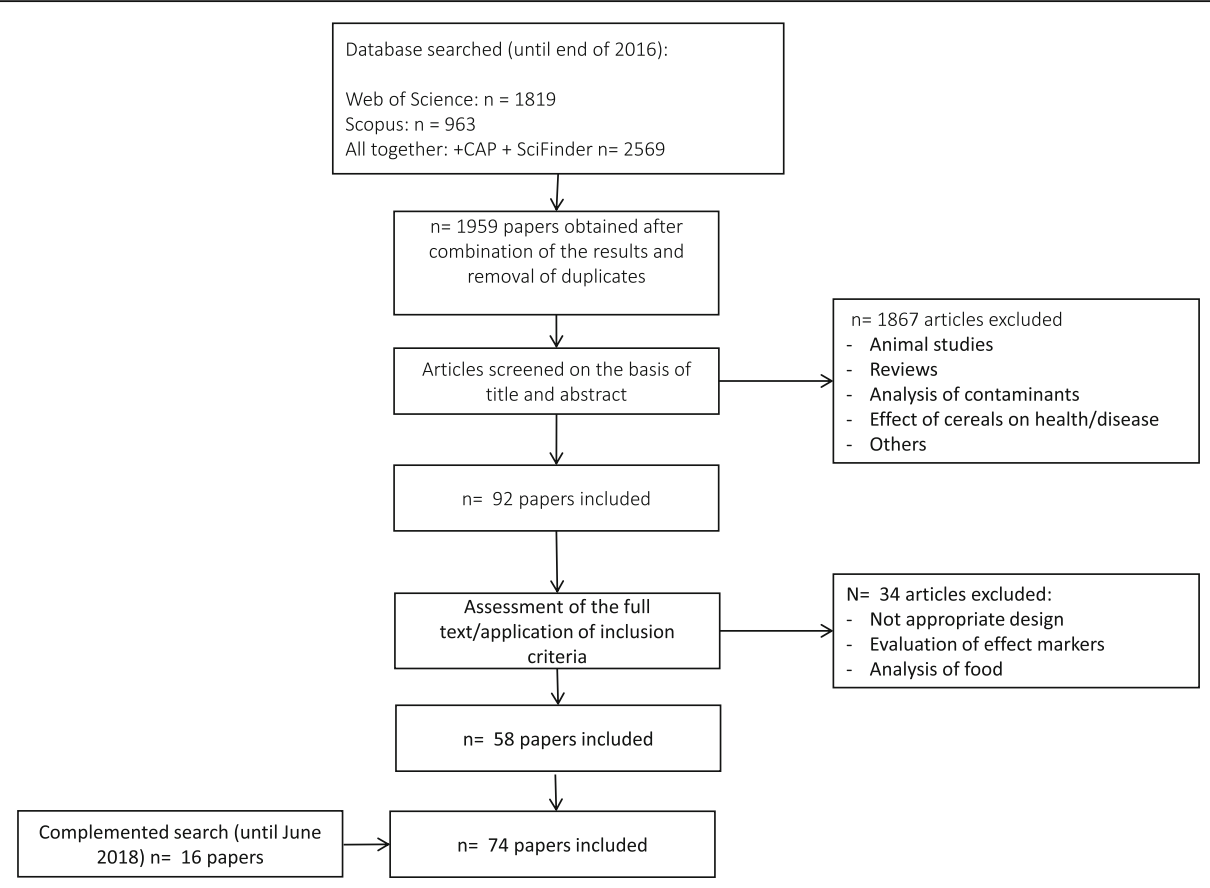

Fig. 1 Flow diagram of study selection

endogenous metabolite reflecting the change in the host metabolic homeostasis evoked by the diet. A comprehensive classification system [36] and a validation scheme for biomarkers of food intake (BFI) has recently been developed by the FoodBall consortium [37]. In this classification, biomarkers were divided into six classes: food compound intake biomarkers (FCIBs), biomarkers of food or food component intake (BFI), dietary pattern biomarkers (DPBs), food compound status biomarkers (FCSBs), effect biomarkers, and physiological or health state biomarkers. According to the traditional classification of dietary biomarkers, FIBs can be classified as recovery and concentration biomarkers depending on their characteristic. In the FoodBall classification system, the recovery biomarkers are included in the health state biomarkers. Recovery biomarkers reflect the balance between intake and excretion of a specific chemical component on an absolute scale over a specific time period whereas concentration biomarkers are correlated with intake [38]. Recovery biomarkers represent the best standard and can be used to calibrate other dietary instruments [39]. Sometimes prediction biomarkers are mentioned as a third category, falling in between the recovery biomarkers and concentration biomarkers [40]. Most biomarkers belong to concentration biomarkers [41].

Targeted and untargeted metabolomics approaches for discovery of cereal biomarkers

Following recent advances over the last years, metabolomics has become a fundamental tool to study changes in molecular phenotype caused by molecules inherent to different exposures, including diet, and their interaction with host risk of disease or other outcomes [42, 43]. Generally speaking, biomarkers may be discovered in different sample matrices such as plasma, erythrocytes, urine, adipose tissue, hair, and nail clippings. Each matrix has their challenges, but in general, dietary biomarkers are typically present at lower concentrations in plasma compared with urine, to which many biomarker molecules or metabolites thereof are excreted. Only a few biomarkers are accumulated in adipose tissue and can be detected in hair. For cereals, individual biomarkers that reflect specific cereal foods have been detected in plasma, urine, and adipose tissues and by utilizing chemometric, multivariate tools, there are new possibilities to use combinations of several biomarkers, i.e., biomarker panels, which may improve prediction of outcomes as well as monitoring of compliance or measuring food intake compared with single concentration biomarker [44].

Metabolomics methodologies can be divided into targeted and untargeted approaches [45]. In targeted metabolomics, a defined set of well-characterized and annotated metabolites are analyzed typically in quantitative platforms such as triple quadrupole mass spectrometry (QQQ-MS) utilizing pure chemicals as standards. Targeted metabolite analyses have been used to analyze compounds known or suspected to be putative biomarkers of specific foods, such as odd-numbered alkylresorcinols (whole-grain wheat and rye) [46], even- 
numbered alkylresorcinols (quinoa) [47], avenanthramides and avenacosides (oats) [48, 49]. In contrast, untargeted approaches aim at maximizing the metabolite coverage in a set of biological samples, even though the vast majority of measured metabolic features remain unidentified. A common analytical platform for profiling assays is quadrupole time-of-flight mass spectrometry (QTOF-MS) hyphenated with chromatographic separation in either liquid or gas phase or by NMR, but so far, we are unaware of any study that has used such approach to discover biomarkers of cereal intake. Metabolite features of special interest are then annotated/ identified at a later stage in the analytical pipeline. Inherent to the wide coverage, untargeted approaches are well suited for exploratory biomarker studies, and this approach has been used to mine for dietary exposure biomarkers reflecting total or specific whole-grain intake as well as specific grain-based foods after controlled interventions with specific foods or reported food intakes [50-53]. Targeted and untargeted approaches are complementary to each other and could both be useful to discover and validate dietary biomarkers. A typical workflow involves identification of putative biomarker candidates via an untargeted profiling approach followed by validation of the biomarkers in targeted, quantitative analyses applied preferentially in other study cohorts. Recently, Zhu et al. [54] combined untargeted and targeted metabolomics approaches to discover biomarkers of whole-grain wheat intake in urine samples after intake of whole-grain wheat bread vs refined wheat bread in a kinetic study in 12 subjects. A panel of urinary markers consisting of seven alkylresorcinol metabolites and five benzoxazinoid derivatives as specific biomarkers, along with five phenolic acid derivatives were suggested to reflect whole-grain wheat intake. Panels of biomarkers of whole grain, refined grain, or fractions of specific grains appear promising but remain to be evaluated in larger studies.

\section{Biomarkers of whole grains, cereal fractions, and specific foods}

To date, only a few specific biomarkers have been suggested for different whole grains, bran fractions, or foods thereof and currently no biomarkers have been suggested for refined grains (Table 1). We are not aware of any compounds that specifically reflect total whole-grain intake from all cereals but a few compounds that are exclusively found in specific whole grains or fractions of specific grains do exist. For example, avenanthramides are only found in oats among cereals and odd-numbered alkylresorcinols are present in wheat, rye, and barley, with specific homolog profiles for the different grains mentioned. The concentrations of these molecules or their metabolites in plasma and urine have been suggested and used as biomarkers of intake (Table 1). Moreover, specific benzoxazinoids and their metabolites in plasma and/or urine have already been shown to be specific to wheat and rye (Table 1). In some cases, molecules that discriminate high vs low whole grain or bran intake vs refined grain after controlled or reported intake have been discovered in plasma and/or urine as metabolites, but they appear not to be specific. For example, different cinnamic acids such as ferulic acid derivatives are abundant in plasma and urine after wheat and rye bran consumption (Table 1). These molecules are found in high contents, mainly bound to the dietary fiber complex primarily in the bran, but are liberated by microbiota in the large intestine and absorbed [81, 91, 92]. They are not unique to any particular grain, they will appear as plasma biomarkers that differentiate between high vs low consumers of whole grain or wheat bran and rye consumers. Since these molecules are present in various other foods and, therefore, not specific to whole-grain intake, they are of limited use as specific dietary biomarkers.

\section{Alkylresorcinols and their metabolites-biomarkers of whole-grain wheat and rye intake}

Alkylresorcinols comprise a group of phenolic lipids that have been suggested and evaluated as specific biomarkers of wheat and rye intake [100]. They are also found in small amounts in barley [46]. Alkylresorcinols are located in the waxy cuticula in between the seed and fruit coats of wheat and rye and are therefore a specific biomarker of bran from these cereals in studies where other whole grains are not consumed [100]. However, bran per se is not often consumed but is usually consumed as whole grain. A large number of studies have been undertaken to evaluate alkylresorcinols as biomarkers after self-reported intake or controlled dietary interventions with different whole-grain wheat and rye products (Table 1). The results suggest that alkylresorcinols can be used as biomarkers of whole-grain wheat and rye, irrespective of food in what food matrix they are present in [19, 29, 32].

Also, alkylresorcinol metabolites in urine and plasma have been suggested to reflect the intake of whole-grain wheat and rye $[101,102]$. Due to their unique presence in the outer parts of wheat and rye grains, alkylresorcinols (AR) are present not only in bran, but also in whole grain (due to the presence of bran), but only in minute amounts in refined flour [100]. Since alkylresorcinols are not degraded in food processing, they will appear in quantities related to the amount of specific whole grain and bran consumed.

The sum of dominant alkylresorcinol homologs with alkyl-chains in the range 17-25 carbon atoms in plasma, i.e., total plasma alkylresorcinol concentration, reflects 
Table 1 Studies reporting candidate biomarkers for cereal food intake

\begin{tabular}{|c|c|c|c|c|c|c|}
\hline $\begin{array}{l}\text { Discriminating } \\
\text { metabolites/candidate } \\
\text { biomarkers }\end{array}$ & Dietary factor & Study design & $\begin{array}{l}\text { Number } \\
\text { of } \\
\text { subjects }\end{array}$ & $\begin{array}{l}\text { Analytical } \\
\text { method }\end{array}$ & Sample type & $\begin{array}{l}\text { Primary } \\
\text { reference(s) }\end{array}$ \\
\hline \multicolumn{7}{|c|}{ Biomarkers of total whole grain (WG) intake } \\
\hline Total ARs & $\begin{array}{l}\text { WG products } \\
\text { RG products }\end{array}$ & $\begin{array}{l}\text { Intervention study, crossover, } \\
\text { randomized ( } 6 \text { weeks) }\end{array}$ & 30 & GC-MS & Plasma & {$[30]$} \\
\hline ARs & WG cereal & Observational study & 33 & GC-MS & Plasma & {$[55]$} \\
\hline ARs & $\begin{array}{l}\text { WG products } \\
\text { RG products }\end{array}$ & $\begin{array}{l}\text { Intervention study, parallel, } \\
\text { randomized (12 weeks) }\end{array}$ & 50 & LC-MS/MS & Plasma & [56] \\
\hline ARs & $\begin{array}{l}\text { WG diet } \\
\text { RG diet }\end{array}$ & $\begin{array}{l}\text { Intervention study, crossover, } \\
\text { randomized ( } 6 \text { weeks) }\end{array}$ & 33 & LC-MS/MS & Plasma & {$[57]$} \\
\hline$A R$ & $\begin{array}{l}\text { WG products } \\
\text { WG rye } \\
\text { WG bread }\end{array}$ & Observational study & 522 & GC-MS & Plasma & {$[58]$} \\
\hline $\begin{array}{c}\text { ARs } \\
\text { Betaine }\end{array}$ & $\begin{array}{l}\text { WG diet } \\
\text { RG diet }\end{array}$ & $\begin{array}{l}\text { Intervention study, crossover, } \\
\text { randomized ( } 2 \text { weeks) }\end{array}$ & 17 & $\begin{array}{l}\text { GC-MS/ } \\
\text { MS (ARS) } \\
\text { LC-MS/MS } \\
\text { (betaine) }\end{array}$ & Plasma & [59] \\
\hline $\begin{array}{l}\text { Total ARs (C17:0, C19:0, } \\
\text { C21:0, C23:0, C25:0) }\end{array}$ & $\begin{array}{l}\text { WG products } \\
\text { Habitual diet }\end{array}$ & $\begin{array}{l}\text { Intervention study, parallel, } \\
\text { randomized (16 weeks) }\end{array}$ & 316 & GC-MS & Plasma & {$[31]$} \\
\hline $\begin{array}{l}\text { Total AR } \\
\text { AR (C17:0-C25:0) }\end{array}$ & WG products & Observational study & 360 & GC-MS & Plasma & {$[60]$} \\
\hline $\begin{array}{l}\text { AR (C17:0, C19:0, C21:0, } \\
\text { C23:0, C25:0) }\end{array}$ & WG products & Observational study & 407 & LC-MS & Plasma & {$[61]$} \\
\hline AR (C17:0-C25:0) & WG products & Observational study & 20 & GC-MS & Adipose tissue & {$[62]$} \\
\hline $\begin{array}{l}\quad \text { DHBA } \\
\text { DHPPA } \\
\text { DHBA+DHPPA }\end{array}$ & $\begin{array}{l}\text { WG products } \\
\text { Cereal fiber }\end{array}$ & Observational study & 104 & GC-MS & Urine & [63] \\
\hline $\begin{array}{l}\text { DHBA } \\
\text { DHPPA } \\
\text { Total metabolites }\end{array}$ & $\begin{array}{l}\text { WG foods } \\
\text { Cereal fiber }\end{array}$ & Observational study & 66 & GC-MS & Urine & {$[64]$} \\
\hline $\begin{array}{l}\text { DHBA } \\
\text { DHPPA }\end{array}$ & $\begin{array}{l}\text { WG products } \\
\text { Fiber }\end{array}$ & Observational study & 2833 & HPLC & Plasma & {$[65]$} \\
\hline DHPPA & $\begin{array}{l}\text { WG products } \\
\text { WG rye and wheat }\end{array}$ & Observational study & 100 & $\begin{array}{l}\text { HPLC- } \\
\text { CEAD }\end{array}$ & Urine & {$[66]$} \\
\hline $\begin{array}{l}\text { 2-Aminophenol-Slf } \\
\text { HPAA-GlcA } \\
\text { HHPAA } \\
\text { HMBOA-GlcA } \\
\text { HBOA glycoside } \\
\text { HPPA } \\
\text { HMBOA } \\
\text { DHPPA-GlcA } \\
\text { 3,5- } \\
\text { Dihydroxyphenylethanol- } \\
\text { SIf } \\
\text { DHPPTA-Slf } \\
\text { Hydroxybenzoic acid-Slf } \\
\text { Dihydroferulic acid-SIf } \\
\text { Enterolactone-GlcA } \\
\text { Pyrraline } \\
\text { 3-Indolecarboxylic acid- } \\
\text { GlcA } \\
\text { Riboflavin } \\
\text { N-a-Acetylcitrulline } \\
\text { 2,8-Dihydroxyquinoline- } \\
\text { GlcA }\end{array}$ & $\begin{array}{l}\text { White bread } \\
\text { WG bread }\end{array}$ & Observational study & 155 & $\begin{array}{l}\text { HPLC-q- } \\
\text { TOF-MS }\end{array}$ & Urine & {$[67]$} \\
\hline $\begin{array}{l}\text { 5-Nonadecyl-1,3- } \\
\text { benzenediol-GlcA (AR) }\end{array}$ & $\begin{array}{l}\text { WG products, fatty fish and } \\
\text { bilberries diet }\end{array}$ & $\begin{array}{l}\text { Intervention study, parallel, } \\
\text { randomized ( } 12 \text { weeks) }\end{array}$ & 106 & $\begin{array}{l}\text { UHPLC-q- } \\
\text { TOF-MS }\end{array}$ & Plasma & [53] \\
\hline
\end{tabular}


Table 1 Studies reporting candidate biomarkers for cereal food intake (Continued)

\begin{tabular}{|c|c|c|c|c|c|c|}
\hline $\begin{array}{l}\text { Discriminating } \\
\text { metabolites/candidate } \\
\text { biomarkers }\end{array}$ & Dietary factor & Study design & $\begin{array}{l}\text { Number } \\
\text { of } \\
\text { subjects }\end{array}$ & $\begin{array}{l}\text { Analytical } \\
\text { method }\end{array}$ & Sample type & $\begin{array}{l}\text { Primary } \\
\text { reference(s) }\end{array}$ \\
\hline benzenediol-GlcA & Refined wheat bread diet & & & & & \\
\hline $\begin{array}{l}\text { Proline } \\
\text { Ornithine } \\
\text { Arginine }\end{array}$ & $\begin{array}{l}\text { Grain protein-based diet (wheat, } \\
\text { bran, rice, and maize) } \\
\text { Dairy protein-based diet (milk } \\
\text { and milk products, yogurt and } \\
\text { cheese) } \\
\text { Meat protein-based diet (pork, } \\
\text { beef, and chicken) }\end{array}$ & $\begin{array}{l}\text { Intervention study, crossover, } \\
\text { randomized (1 week) }\end{array}$ & 100 & $\begin{array}{l}\text { LC- } \\
\text { tripleQ- } \\
\text { MS }\end{array}$ & Plasma & {$[68]$} \\
\hline \multicolumn{7}{|c|}{ Biomarkers of whole-grain wheat and rye intake } \\
\hline Total ARs & $\begin{array}{l}\text { WG rye bread and WG wheat } \\
\text { bread } \\
\text { Gluten-free diet }\end{array}$ & $\begin{array}{l}\text { Intervention study, parallel ( } 2 \\
\text { weeks) }\end{array}$ & 9 & GC-MS & $\begin{array}{l}\text { Plasma and } \\
\text { erythrocyte } \\
\text { membranes }\end{array}$ & {$[69]$} \\
\hline Total ARs & $\begin{array}{l}\text { WG rye and wheat } \\
\text { Cereal fiber }\end{array}$ & Observational study & 51 & GC-MS & Plasma & {$[70]$} \\
\hline $\begin{array}{l}\text { Total ARs } \\
\text { AR C17:0, C19:0, C21:0, } \\
\text { C23:0, C25:0 } \\
\text { Enterolactone }\end{array}$ & $\begin{array}{l}\text { Rye bread } \\
\text { Wheat bread }\end{array}$ & $\begin{array}{l}\text { Intervention study, crossover, } \\
\text { randomized (8 weeks) }\end{array}$ & 39 & GC-MS & Plasma & {$[71]$} \\
\hline $\begin{array}{l}\text { Total AR (C17:0, C19:0, } \\
\text { C21:0, C23:0, C25:0) } \\
\text { AR C17:0/C21:0 } \\
\text { Enterolactone }\end{array}$ & $\begin{array}{l}\text { WG wheat } \\
\text { WG rye crisp bread }\end{array}$ & $\begin{array}{l}\text { Intervention study, crossover, } \\
\text { randomized ( } 1 \text { week) }\end{array}$ & 15 & GC-MS & $\begin{array}{l}\text { Plasma (AR) } \\
\text { erythrocyete } \\
\text { membrane (AR) } \\
\text { Lipoproteins (AR) } \\
\text { Serum } \\
\text { (enterolactone) }\end{array}$ & [69] \\
\hline $\begin{array}{l}\text { Total ARs (C17:0-C25:0) } \\
\text { AR C17:0/C21:0 }\end{array}$ & $\begin{array}{l}\text { Nordic diet (rich in WG rye and } \\
\text { wheat) } \\
\text { Control diet }\end{array}$ & $\begin{array}{l}\text { Intervention study, parallel, } \\
\text { randomized (18-24 weeks) }\end{array}$ & 158 & GC-MS & Plasma & [32] \\
\hline $\begin{array}{l}\text { Total ARs } \\
\text { AR C17:0/C21:0 }\end{array}$ & $\begin{array}{l}\text { WG wheat } \\
\text { RG wheat }\end{array}$ & $\begin{array}{l}\text { Intervention study, parallel, } \\
\text { randomized ( } 12 \text { weeks) }\end{array}$ & 72 & LC-MS/MS & Plasma & {$[72]$} \\
\hline $\begin{array}{l}\text { DHBA } \\
\text { DHPPA }\end{array}$ & $\begin{array}{l}\text { Rye bread } \\
\text { Bread fiber }\end{array}$ & Observational study & 122 & $\begin{array}{l}\text { HPLC- } \\
\text { CEAD }\end{array}$ & $\begin{array}{l}\text { Urine } \\
\text { Plasma }\end{array}$ & {$[73]$} \\
\hline $\begin{array}{l}\text { DHBA } \\
\text { DHPPA }\end{array}$ & $\begin{array}{l}\text { Rye bread with plant sterols } \\
\text { Rye bread }\end{array}$ & $\begin{array}{l}\text { Intervention study, parallel, } \\
\text { randomized ( } 4 \text { weeks) }\end{array}$ & 68 & $\begin{array}{l}\text { HPLC- } \\
\text { CEAD }\end{array}$ & Plasma & {$[74]$} \\
\hline $\begin{array}{l}\text { DHBA } \\
\text { DHPPA }\end{array}$ & Rye bread & $\begin{array}{l}\text { Intervention study } \\
\text { (postprandial trial) }\end{array}$ & 15 & $\begin{array}{l}\text { HPLC- } \\
\text { CEAD }\end{array}$ & Urine & {$[75]$} \\
\hline $\begin{array}{l}\text { DHBA } \\
\text { DHPPA }\end{array}$ & Rye bread & $\begin{array}{l}\text { Intervention study } \\
\text { (postprandial trial) }\end{array}$ & 15 & $\begin{array}{l}\text { HPLC- } \\
\text { CEAD }\end{array}$ & Plasma & {$[76]$} \\
\hline $\begin{array}{l}\text { DHBA } \\
\text { DHPPA }\end{array}$ & Rye & Observational study & 60 & $\begin{array}{l}\text { HPLC- } \\
\text { CEAD }\end{array}$ & $\begin{array}{l}\text { Urine } \\
\text { Plasma }\end{array}$ & {$[77]$} \\
\hline $\begin{array}{l}\text { DHBA } \\
\text { DHPPA }\end{array}$ & WG rye and wheat & Observational study & 52 & GC-MS & Urine & {$[78]$} \\
\hline $\begin{array}{l}\quad \text { DHBA } \\
\text { DHBA glycine } \\
\text { DHPPA } \\
\text { DHPPTA }\end{array}$ & $\begin{array}{l}\text { RG wheat bread, WG wheat } \\
\text { bread }\end{array}$ & $\begin{array}{l}\text { Intervention study } \\
\text { (postprandial trial) }\end{array}$ & 12 & $\begin{array}{l}\text { LC-MS } \\
\text { HPLC- } \\
\text { CEAD }\end{array}$ & Urine & {$[79]$} \\
\hline $\begin{array}{l}\text { HHPAA-GlcA } \\
\text { HHPAA-SIf } \\
\text { HPAA-SIf } \\
\text { HBOA-GlcA } \\
\text { Phenylacetylglutamine } \\
\text { derivative } \\
\text { Creatinine } \\
\text { N-feruloylglycine-Slf }\end{array}$ & WG rye foods & Intervention study (8 weeks) & 33 & FIE-MS & Urine & {$[80]$} \\
\hline $\begin{array}{l}\text { DHFA } \\
\text { Ferulic acid }\end{array}$ & $\begin{array}{l}\text { WG wheat products } \\
\text { Refined wheat products }\end{array}$ & $\begin{array}{l}\text { Intervention study, parallel, } \\
\text { randomized (8 weeks) }\end{array}$ & 80 & $\begin{array}{l}\text { HPLC-MS/ } \\
\text { MS }\end{array}$ & $\begin{array}{l}\text { Urine } \\
\text { Serum } \\
\text { Feces }\end{array}$ & {$[81]$} \\
\hline
\end{tabular}


Table 1 Studies reporting candidate biomarkers for cereal food intake (Continued)

\begin{tabular}{|c|c|c|c|c|c|c|}
\hline $\begin{array}{l}\text { Discriminating } \\
\text { metabolites/candidate } \\
\text { biomarkers }\end{array}$ & Dietary factor & Study design & $\begin{array}{l}\text { Number } \\
\text { of } \\
\text { subjects }\end{array}$ & $\begin{array}{l}\text { Analytical } \\
\text { method }\end{array}$ & Sample type & $\begin{array}{l}\text { Primary } \\
\text { reference(s) }\end{array}$ \\
\hline $\begin{array}{l}\text { 3,5- } \\
\text { Dihydroxyhydrocinamic } \\
\text { acid sulfate } \\
\text { Ascorbic acid } \\
\text { 2-Aminophenol-Slf } \\
\text { Nonanedioic acid } \\
\text { DHPPA-GlcA } \\
\text { Indolylacryloylglycine } \\
\text { Enterolactone-GlcA } \\
\text { DHPPA-Slf } \\
\text { Ferulic acid-4-SIf } \\
\text { 2,4-Dihydroxy-1,4- } \\
\text { benzoxazin-3-one-SIf } \\
\text { 3,5- } \\
\text { Dihydroxyphenylethanol- } \\
\text { SIf } \\
\text { 1,3,4,5- } \\
\text { Tetrahydroxycyclohexane- } \\
\text { 1-carboxylic acid } \\
\text { Others non-identified } \\
\text { metabolites }\end{array}$ & $\begin{array}{l}\text { WG rye bread } \\
\text { Refined wheat bread }\end{array}$ & $\begin{array}{l}\text { Intervention study, crossover, } \\
\text { randomized ( } 4 \text { weeks) }\end{array}$ & 20 & $\begin{array}{l}\text { UPLC-q- } \\
\text { TOF-MS }\end{array}$ & Urine & {$[50]$} \\
\hline \multicolumn{7}{|c|}{ Biomarkers of specific fractions of foods of wheat and rye intake } \\
\hline $\begin{array}{l}\text { Total ARs } \\
\text { AR (C17:0, C19:0, C21:0, } \\
\text { C23:0, C25:0) }\end{array}$ & $\begin{array}{l}\text { WG grains } \\
\text { Cereal fiber } \\
\text { Bran } \\
\text { Total fiber } \\
\text { Legume fiber }\end{array}$ & Observational study & 165 & GC-MS & Plasma & [82] \\
\hline $\begin{array}{c}\text { Total ARs } \\
\text { AR C17:0/C21:0 }\end{array}$ & Cereal fiber & Observational study & 2845 & GC-MS & Plasma & {$[83]$} \\
\hline $\begin{array}{l}\text { Total ARs } \\
\text { AR C17:0/C21:0 }\end{array}$ & Cereal fiber & Observational study & 2744 & GC-MS & Plasma & {$[84]$} \\
\hline $\begin{array}{c}\text { Total AR } \\
\text { AR C17:0/C21:0 }\end{array}$ & $\begin{array}{l}\text { Rye WG/bran } \\
\text { Refined wheat }\end{array}$ & $\begin{array}{l}\text { Intervention study, crossover, } \\
\text { randomized ( } 6 \text { weeks) }\end{array}$ & 17 & GC-MS & Plasma & {$[85]$} \\
\hline $\begin{array}{l}\text { AR C23:0 } \\
\text { AR C25:0 } \\
\text { AR C17:0/C21:0 }\end{array}$ & Rye bran flakes & $\begin{array}{l}\text { Intervention study } \\
\text { (postprandial trial) }\end{array}$ & 6 & GC-MS & Plasma & {$[86]$} \\
\hline $\begin{array}{l}\quad \text { ARs (plasma) } \\
\text { DHBA (urine) } \\
\text { DHPPA (urine) }\end{array}$ & Cereal fiber & Observational study & 56 & $\begin{array}{l}\text { HPLC- } \\
\text { CEAD }\end{array}$ & $\begin{array}{l}\text { Urine } \\
\text { Plasma }\end{array}$ & {$[87]$} \\
\hline $\begin{array}{l}\text { DHBA } \\
\text { DHPPA }\end{array}$ & Cereal fiber & Observational study & 56 & $\begin{array}{l}\text { HPLC- } \\
\text { CEAD }\end{array}$ & Plasma & {$[88]$} \\
\hline $\begin{array}{l}\text { HPAA-SIf } \\
\text { HHPAA-SIf }\end{array}$ & $\begin{array}{l}\text { WG sourdough rye bread } \\
\text { White bread with native } \\
\text { unprocessed rye bran } \\
\text { White bread with bioprocessed } \\
\text { rye bran } \\
\text { White bread }\end{array}$ & $\begin{array}{l}\text { Intervention study, crossover, } \\
\text { randomized (postprandial } \\
\text { trial) }\end{array}$ & 12 & $\begin{array}{l}\text { LC-q-TOF- } \\
\text { MS }\end{array}$ & Plasma & {$[52]$} \\
\hline $\begin{array}{l}\text { 2,6-dihydroxybenzoic } \\
\text { acid } \\
\text { 2-aminophenol-Slf }\end{array}$ & $\begin{array}{l}\text { High fiber diet } \\
\text { Low fiber diet }\end{array}$ & $\begin{array}{l}\text { Intervention study, crossover, } \\
\text { randomized ( } 5 \text { weeks) }\end{array}$ & 25 & $\begin{array}{l}\text { UPLC-q- } \\
\text { TOF-MS }\end{array}$ & Plasma & {$[89]$} \\
\hline Ferulic acid & $\begin{array}{l}\text { Rye bran bread } \\
\text { Inert wheat bran }\end{array}$ & $\begin{array}{l}\text { Intervention study, crossover, } \\
\text { randomized ( } 6 \text { weeks) }\end{array}$ & 18 & HPLC & Urine & {$[90]$} \\
\hline $\begin{array}{l}\quad \text { Ferulic acid } \\
\text { Vanillic acid } \\
\text { Sinapic acid } \\
\text { 3,4-Dimethoxybenzoic } \\
\text { acid } \\
\text { Phenylpropionic acid } \\
\text { 3-Hydroxyphenylpropionic } \\
\text { acid }\end{array}$ & $\begin{array}{l}\text { Whole wheat bread with } \\
\text { bioprocessed bran } \\
\text { Whole wheat bread with native } \\
\text { bran }\end{array}$ & $\begin{array}{l}\text { Intervention study, crossover, } \\
\text { randomized (postprandial } \\
\text { trial) }\end{array}$ & 8 & $\begin{array}{l}\text { GCxGC-q- } \\
\text { TOF-MS }\end{array}$ & $\begin{array}{l}\text { Urine } \\
\text { Plasma }\end{array}$ & {$[91]$} \\
\hline
\end{tabular}


Table 1 Studies reporting candidate biomarkers for cereal food intake (Continued)

\begin{tabular}{|c|c|c|c|c|c|c|}
\hline $\begin{array}{l}\text { Discriminating } \\
\text { metabolites/candidate } \\
\text { biomarkers }\end{array}$ & Dietary factor & Study design & $\begin{array}{l}\text { Number } \\
\text { of } \\
\text { subjects }\end{array}$ & $\begin{array}{l}\text { Analytical } \\
\text { method }\end{array}$ & Sample type & $\begin{array}{l}\text { Primary } \\
\text { reference(s) }\end{array}$ \\
\hline $\begin{array}{l}\text { Ferulic acid } \\
\text { Sinapic acid }\end{array}$ & $\begin{array}{l}\text { White wheat bread with } \\
\text { bioprocessed rye bran } \\
\text { White wheat bread with native } \\
\text { rye bran }\end{array}$ & $\begin{array}{l}\text { Intervention study, crossover, } \\
\text { randomized (postprandial } \\
\text { trial) }\end{array}$ & 15 & GC-MS & Urine & [92] \\
\hline $\begin{array}{l}\text { Indole-2-carboxylic acid } \\
\text { Hydrocinnamic acid } \\
\text { a-tocopherol } \\
\text { Benzoic acid } \\
\text { Cycloartenol } \\
\text { Pantothenic acid } \\
\text { Phenylacetic acid } \\
\beta \text {-sitosterol }\end{array}$ & Heat-stabilized rice bran & $\begin{array}{l}\text { Intervention study, parallel, } \\
\text { randomized ( } 4 \text { weeks) }\end{array}$ & 7 & GC-MS & Feces & [93] \\
\hline \multicolumn{7}{|l|}{ Biomarkers of quinoa } \\
\hline Even-numbered AR & Quinoa & Intervention study & NS & $\begin{array}{l}\text { LC-MS/MS } \\
\text { GC-MS/ } \\
\text { MS }\end{array}$ & Plasma & [94] \\
\hline \multicolumn{7}{|c|}{ Biomarkers dependent on gut microbiota } \\
\hline Enterolactone & $\begin{array}{l}\text { High-fiber bread } \\
\text { Fiber } \\
\text { Fruits and berries }\end{array}$ & Observational study & 1099 & TRFIA & Plasma & [95] \\
\hline Enterolactone & WG products & Observational study & 1889 & TRFIA & Plasma & [95] \\
\hline Enterolactone & $\begin{array}{l}\text { High-fiber rye } \\
\text { High-fiber wheat } \\
\text { Low-fiber foods }\end{array}$ & $\begin{array}{l}\text { Intervention study, crossover, } \\
\text { randomized ( } 4 \text { weeks) }\end{array}$ & 28 & TRFIA & Plasma & [96] \\
\hline Enterodiol & $\begin{array}{l}\text { WG diet } \\
\text { RG diet }\end{array}$ & $\begin{array}{l}\text { Intervention study, crossover, } \\
\text { randomized (12 weeks) }\end{array}$ & 13 & $\begin{array}{l}\text { HPLC- } \\
\text { (EAD) }\end{array}$ & Urine & [97] \\
\hline $\begin{array}{l}\text { Enterolactone } \\
\text { Enterodiol }\end{array}$ & $\begin{array}{l}\text { Quinoa flakes } \\
\text { Corn flakes }\end{array}$ & $\begin{array}{l}\text { Intervention study, parallel, } \\
\text { randomized ( } 4 \text { weeks) }\end{array}$ & 35 & HPLC & $\begin{array}{l}\text { Urine } \\
\text { Serum }\end{array}$ & [98] \\
\hline $\begin{array}{l}\text { Enterodiol (serum) } \\
\text { Enterolactone (serum \& } \\
\text { urine) }\end{array}$ & $\begin{array}{l}\text { Flaxseed } \\
\text { Rice }\end{array}$ & $\begin{array}{l}\text { Intervention study, parallel (6 } \\
\text { weeks) }\end{array}$ & 27 & HPLC & $\begin{array}{l}\text { Urine } \\
\text { Serum }\end{array}$ & [99] \\
\hline
\end{tabular}

Abbreviations: $A R$ alkylresorcinols, CEAD coulometric electrode array detection, $D H B A$ 3,5-dihydroxy-benzoic acid, DHBA glycine 2-(3,5-dihydroxybenzamido)acetic acid, DHFA dihydroferulic acid, DHPPA 3-(3,5-dihydroxyphenyl)-1-propanoic acid, DHPPTA 5-(3,5-dihydroxyphenyl)pentanoic acid, FIE flow infusion electrosprayionization, GC gas chromatography, GCXGC two-dimensional GC, GlCA glucuronide, HBOA 2-Hydroxy-1,4-benzoxazin-3-one, HHPAA 2-hydroxy-N-(2-hydroxyphenyl) acetamide, HPAA N-(2-hydroxyphenyl) acetamide, HPLC high-performance liquid chromatography, LC liquid chromatography, MS mass spectrometry, MS/MS tandem mass spectrometry, NS not specified, $q$-TOF quadrupole time-of-flight, $R G$ refined-grain, SIf sulfate, TRFIA time-resolved fluoroimmunoassay, WG whole-grain

total whole-grain wheat and rye intake in a dosedependent manner [85]. The alkylresorcinol homolog profile is specific for common wheat, durum wheat, and rye, and the ratio of two specific alkylresorcinol homologs $(\mathrm{C} 17: 0 / \mathrm{C} 21: 0)$ can be used as a biomarker of the intake of whole-grain rye to whole-grain wheat intake, since the ratio is always 1.0 in whole-grain rye-based foods, 0.1 in common wheat and 0.01 in durum wheat $[30,31,71,85,103,104]$. Plasma alkylresorcinols have a rather short apparent elimination half-life (4-6h) and thus reflect medium- to long-term whole-grain wheat and rye intake in populations with stable and frequent intake $[29,105]$ but are less suitable in populations where intake is less frequent $[82,106]$.

Alkylresorcinols are metabolized by CYP450dependent metabolism, which causes insertion of an $\mathrm{OH}$-group at the end of the alkyl-chain, followed by subsequent oxidation into a $\mathrm{COOH}$-group. This derivative then undergoes beta-oxidation, where the side-chain is degraded by stepwise removal of $\mathrm{C}_{2} \mathrm{H}_{5}$ per cycle, generating free and conjugated 1,3-dihydroxy-benzoic acid (DHBA) and 3-(1,3-dihydroxyphenyl)-1-propionic acid (DHPPA) as the main end products [102]. Urinary excretion of DHBA and DHPPA has been shown similar validity as plasma AR concentrations in free-living subjects with high and frequent intake [64, 87]. As expected, spot urine samples fluctuated more day to day and were somewhat less correlated with estimated intake compared with 24-h collections [63, 64, 66]. Some studies report that DHBA and DHPPA are also present in urine after intake of food compounds not derived from cereals [34]. This could have implications for the use of DHBA and DHPPA as biomarkers of whole-grain wheat and rye intake, particularly in populations with low intake. 
However, intakes have been correlated with levels of DHBA and DHPPA in urine from the US population where the intake of whole grain is small [63].

Recently, new alkylresorcinol metabolites (DHBA-glycine, DHPPTA, DHCA) were detected in urine from mice and/or humans and their half-lives were suggested to be longer than that of previously identified alkylresorcinol metabolites [50, 79, 101]. These biomarkers were evaluated in free-living Swedish men and women and results showed that DHPPTA and DHCA determined in single 24-h urine excretions had excellent reproducibility (ICC $=0.63$ for both $)$ and good relative validity $(r=0.40-$ 0.65 ), and thus could be useful as long-term biomarkers of whole-grain wheat and rye intake [102]. However, 24$\mathrm{h}$ urine collections are typically not available in large scale epidemiologic studies, but spot urine samples may be more readily available. It is therefore highly relevant to asses if these biomarkers analyzed in spot urine samples remain useful as biomarkers of wheat/rye wholegrain intake $[63,107]$.

\section{Benzoxazinoids-potential biomarkers of whole-grain wheat and rye as well as for sourdough rye}

Benzoxazinoids is another group of compounds that comprise specific derivatives almost exclusively found in wheat and rye grains. These compounds have been originally described in the context of the defense mechanism of certain plant species including rye, wheat, and maize [108]. Nowadays, they are studied as part of the dietary compound repertoire related in particular to whole-grain wheat and rye. Dihm et al. [109] conducted a detailed study where major benzoxazinoid compounds were quantified in various grain-based products namely commercial flours (whole-grain wheat flour, coarse-rye flour, fine-rye flour, refined-wheat flour, graham flour, quinoa flour, teff flour) and 20 commercial breads from Scandinavia, as well as 3 traditionally home-baked breads. The highest amount of benzoxazinoid compounds were found in flour from fine-rye flour $(3.6 \mathrm{mg} / \mathrm{g}$ dry weight), whereas two Finnish rye breads had the highest amount among the bread products $(2.3-3.3 \mathrm{mg} /$ g dry weight). In all cases, the double-hexose conjugated 2,4-dihydroxy-1,4-benzoxazin-3-one (DIBOA) was the main benzoxazinoid metabolite.

The concentrations of specific benzoxazinoids and their metabolites in biofluids are largely affected by factors other than the whole-grain content, such as processing. For example, the double-hexose conjugated compounds abundant in flour are easily degraded during sourdough fermentation [110]. Interestingly, in the study by Dihm et al., [109] the level of double-hexose conjugated forms of benzoxazinoids was very high in two commercial Finnish breads, which further highlights the fact that the processing method can remarkably affect the chemical composition and dietary intake thereafter [108]. Few studies have investigated the concentration of benzoxazinoids in plasma and urine samples after whole-grain consumption (Table 1). Hanhineva et al. showed modest correlations between estimated wholegrain rye intake and benzoxazinoid in 24-h urine, but the levels were found to fluctuate extensively over a period of 2-3 months [51]. Other studies have shown increased plasma, urine, and tissue benzoxazinoid concentrations after intake of benzoxazinoid-rich foods from wheat and rye [50,52]. A particular metabolite derived from benzoxazinoids via metabolism by the gut microbiota, and conjugation in the liver, is aminophenol sulfate. This compound has been reported in urine after intake of various bread products. Therefore, it appears that native benzoxazinoids present in the grains undergo massive conformational changes during technological processing a gut fermentation, as well as endogenous metabolism, and the kinetics as well as particular chemical conversions of the whole pathway are not yet known. Thus, more studies are needed to evaluate the feasibility of individual benzoxazinoids and their metabolites as biomarkers of WG intake.

Studies suggest that dietary benzoxazinoids are converted into phenylacetamides (2-hydroxy- $N$-(2-hydroxyphenyl)acetamide (HHPAA) and $N$-(2hydroxyphenyl)acetamide (HPAA)) that are detectable in urine and plasma after consumption of a meal rich in whole grains [52]. Steffensen et al. investigated the concentration of different benzoxazinoids after intake of benzoxazinoid-rich foods from rye (flakes, porridge, and breads) in plasma, urine, and in prostate tissue in men with prostate cancer [111]. The overall finding was that benzoxazinoids increased in all matrices after highbenzoxazinoid-based rye foods, but different forms dominated in different matrices and varied between subjects. Beckmann et al. [80] used flow infusion electrospray mass spectrometry (MS) to profile metabolites in urine from participants who reported high intakes of rye flakes, rye pasta, or total whole-grain rye products, but they could not find any discriminative metabolites compared with subjects wash-out samples. However, they observed discrimination in urine samples from participants who reported high whole-grain sourdough rye bread consumption. They found that benzoxazinoid lactam 2-hydroxy-1,4-benzoxazin-3-one and hydroxylated phenyl acetamide derivatives were higher after sourdough rye bread consumption and that these molecules may be candidate biomarkers of such foods. However, as noted by Hanhineva et al. [52], bioprocessing such as baking that involves microbial metabolism (e.g., sourdough fermentation) has a central role in modulating the phytochemical content in whole-grain and bran-rich breads and it is likely that differences in the processes 
and inclusion of different starters etc. may cause variation in suggested biomarkers. Thus, biomarkers that may be valid for one type of product may not be universal to all.

Only very few studies have investigated whether benzoxazinoids or their metabolites could be used as biomarkers of whole-grain wheat and rye intake or for specific foods, such as sourdough-fermented rye bread. In one study, their levels in urine samples were well correlated with estimated whole-grain rye intake [53], but they were found to fluctuate considerably in urine samples taken 2-3 months in between, probably due to short half-lives. Further studies are needed to validate benzoxazinoids as biomarkers in both controlled feeding-trials and in observational studies. Correlations with estimated intakes as well as stability over time need to be estimated to assess their usefulness as biomarkers. Indeed, the involvement of the gut microbiota in benzoxazinoids metabolism necessitates further study to establish which microorganisms or groups of microorganisms may be involved. Recent observations that certain metabotypes (e.g., urolithin metabotypes) determined by gut microbiota metabolism of other polyphenols may not be stable over time but change with age, habitual dietary intake, obesity, disease state, etc. suggest that gut microbiota community structure and metabolic output are closely linked but not fixed for a given individual [112]. This also has implications for the validity of small phenolic acids as biomarkers of intake, if their production from benzoxazinoids for example, changes as gut microbiota composition changes. Similarly, since we still know little about how specific polyphenol-derived small phenolic acids impact human physiology, the significance of pliable metabotypes in terms of human health remains to be determined.

\section{Biomarkers of whole-grain oat intake}

Until recently, there have been no biomarker candidates of whole-grain oat intake. Oats contains two classes of unique compounds: avenanthramides (AVAs) and steroidal saponins. AVAs are substituted $N$-cinnamoylanthranilic acids consisting of anthranilic acid and cinnamic acid moieties. To date, 25 AVAs which differ in the substitution patterns of two moieties have been identified in oats; some at very low concentrations [113]. The most common avenanthramides are AVA-A (2p), AVA-B (2f), and AVA-C (2c) and differ only by a single moiety on the hydroxycinnamic acid ring. Several studies have evaluated the uptake of avenanthramides in humans and these studies found that different avenanthramides show different, but consistently low, bioavailability in humans. Chen et al. [114] reported that serum levels of AVA 2p, 2f, and $2 \mathrm{c}$ reach a peak $2 \mathrm{~h}$ after consumption of an AVA-enriched mixture (AEM) with a gradual return to base-line within $10 \mathrm{~h}$. Recently, Zhang et al. [115] showed for the first time that AVAs were bioavailable in humans, after consumption of cookies based on regular oat flour. Previous studies had used AVA-enriched fractions or extracts [115]. AVA-B has the slowest elimination rate and the longest half-life compared to AVA-A and AVA-C. The half-lives were in the range 2-5hours [116]. AVAs like other phenolic compounds are extensively metabolized. Walsh et al. [116] fed 12 subjects with muffins with oat bran enriched in AVA and investigated potential metabolites of AVAs in plasma. They identified two methylated AVAs but did not detect any sulfate- or glucuronide conjugates.

Schär et al. [117] studied the excretion of phenolic acids and avenanthramides in urine samples among seven subjects after consumption of $60 \mathrm{~g}$ of oat bran compared to a control diet low in phenolic compounds. In total, 30 compounds were higher in urine up to $8 \mathrm{~h}$ after oat bran consumption. Vanillic acid, 4- and 3hydroxyhippuric acids, and sulfate-conjugates of benzoic and ferulic acids were the major compounds excreted. Sang et al. [49] investigated whether AVAs and their metabolites could be used as exposure markers for whole-grain oat intake. They identified a reduction of the double bond in the cinnamic acid unit and cleavage of the amide bond as the major metabolic pathways of AVAs, that the double bond reduced metabolites ( $\mathrm{DH}-$ AVAs) were derived from gut microbiota. Excretion in urine suggested that the DH-AVAs had longer half-life and that the combination of AVAs and DH-AVAs may better reflect long-term intake and may jointly be used as biomarkers of whole-grain oat intake. However, not all participants produced DH-AVAs which suggest that microbiota is an important determinant that may need to be taken into account. However, as with other cereals, we still know little about which bacteria or groups of bacteria are involved, and how their production of these metabolites changes with age, sex, health status, or xenobiotic (e.g., drug) exposure.

Oat contains two unique steroid glycosides, avenacoside-A (AVE-A) and AVE-B [113, 118]. AVE-A and $-B$ are present in high concentrations in oat bran products with a total content of AVE-A and -B that varies from 304 to $443.0 \mathrm{mg} / \mathrm{kg}$ [118]. To our knowledge, only one study has been reported where the metabolic fate of AVEs has been investigated [48]. Wang et al. analyzed AVE-A and B in urine from 12 individuals during $48 \mathrm{~h}$ after a single dose of oat bran and they also assessed the potential impact of the human gut microbiota. The aim was to evaluate the potential of these molecules as putative biomarkers of whole-grain oat intake. The concentrations of AVE-A and -B increased rapidly after oat bran intake. The average apparent half- 
lives were $4.5 \mathrm{~h}$ and 6.2 for AVE-A and -B, respectively. Deglycosylation was identified as the major metabolic path for AVE-A and -B metabolism in experiments where pure AVE-A and -B were incubated with human fecal samples. Both human and mice gut microbiota metabolized AVE-A and -B in a similar way and 3 metabolites of AVE-A and 5 metabolites of AVE-B were detected from both man and mice [48]. The total 24-h urinary recovery of AVE-A and $-B$ was $<5 \%$ of ingested dose. The influence of gut microbiota on AVE-A and -B may affect their validity as biomarkers, but further studies need to be conducted to evaluate this.

Both avenanthramides, avenacosides, and their metabolites may have potential as short-to-medium-term biomarkers of oat intake, since they are not found in other commonly consumed foods. However, they show low bioavailability and rapid metabolism which is partly dependent on gut microbiota. This probably affects their potential as biomarkers of oat intake, but validation studies are needed to confirm biomarker status [34].

\section{Biomarkers of quinoa intake}

Quinoa is a pseudocereal typically grown in the Andes, but consumption is expanding especially in Europe mainly because of its nutritional profile and its use as a gluten-free alternative to cereal grains. Moreover, quinoa is not short in lysine, which increases the bioavailability of its amino acids, and could increase the nutritional value of gluten-free diets $[119,120]$. Recently, Ross et al. discovered and profiled alkylresorcinols in 17 commercial samples of quinoa [47]. Interestingly, the authors found a surprising AR profile in quinoa samples, with about 30 alkylresorcinol derivatives including odd-, even-, and branched-chain alkylresorcinols as well as methyl-alkylresorcinols. The total AR contents in the quinoa samples were $58 \pm 16 \mu \mathrm{g} / \mathrm{g}$ (AR), $182 \pm 52 \mu \mathrm{g} / \mathrm{g}$ (branched-chain alkylresorcinols) and $136 \pm 40 \mu \mathrm{g} / \mathrm{g}$ (methyl-alkylresorcinols) [47]. These values were much lower than those reported in rye and wheat but in a similar magnitude as those quantified in barley [121]. Some of the alkylresorcinol homologs in quinoa are also present in other cereal species but the unique alkylresorcinol homolog composition profile with even-numbered alkylresorcinol homologs in quinoa allows its discrimination from those alkylresorcinol derived from wheat, rye, and barley [122]. It should be noted that some of the compounds were identified for the first time in nature. Among the even-numbered alkylresorcinol homologs, C18:0, C20:0, C22:0, and C24:0 are commonly present in quinoa but not in other cereals [47]. In order to evaluate whether even-numbered alkylresorcinols in plasma could be used as biomarkers of quinoa intake, Ross et al. [47] applied a liquid chromatography tandem mass spectrometry (LC-MS/MS) method to identify and quantify the even-chained alkylresorcinols in plasma from a volunteer $12 \mathrm{~h}$ after consuming $100 \mathrm{~g}$ (uncooked weight) of white quinoa. The authors showed that the concentrations of these metabolites were higher in plasma after quinoa consumption. Alkylresorcinol C22:0 had previously been described in volunteers following a crossover intervention with a gluten-free diet, possibly due to quinoa intake [122]. In conclusion, since quinoa appears to be an exclusive source of the even-chain alkylresorcinols and because these compounds have been detected and quantified in plasma samples following quinoa intake, it is feasible to propose these compounds as biomarkers of quinoa intake. However, validation studies to assess halflife, dose response, reproducibility, and validity under controlled intake and under free-living condition are needed.

\section{Biomarkers of rice intake}

Very few studies have been reported where putative biomarkers of rice intake were explored. Guertin et al. [123], analyzed baseline serum samples from 502 participants in the Prostate, Lung, Colorectal, and Ovarian (PLCO) Cancer Screening Trial with LC-MS/MS and gas chromatography mass spectrometry (GC-MS). They detected 412 known metabolites and correlated these to different food intakes, reported by FFQ, including rice intake. Among investigated metabolites, only docosahexaenoic acid (DHA) correlated significantly with rice intake, and this was likely due to confounding by fish intake. $\mathrm{Li}$ et al. [124] analyzed the plasma metabolome in 38 children after 4-week intake of rice bran in one arm of a study to investigate its impact on cholesterol concentrations in plasma. The authors analyzed 854 metabolites in plasma and about 300 were also found in the rice bran food metabolome. Rice bran metabolites detected with high relative abundance in plasma included methionine sulfone, alpha-hydroxycaproate, linoleoyllinolenoyl-glycerol, palmitoyl-linolenoylglycerol, pyridoxal, 2-hydroxyhippurate, salicylate, gammaglutamylglutamate, gamma-glutamylthreonine, hypoxanthine, and dihydroorotate. However, it is unclear to what extent these metabolites, separately or in combination, would specifically reflect rice bran intake.

In another study [125], the same group applied GCMS-based metabolomics on stool samples from 19 colorectal cancer survivors who were fed heated rice bran or control for a period of 4 weeks. They found 39 metabolites that were higher after rice bran intake compared with baseline and which, at the same time, overlapped with the rice bran metabolome. These metabolites included lipid compounds, tryptophan metabolites, flavonoids, and B-vitamins, among other molecules. Although the authors suggest that rice bran-derived phytochemicals in plasma and stool samples may be used as 
biomarkers of rice bran intake, most metabolites are likely too unspecific to be used as specific biomarkers of rice bran intake. Further studies are needed to find biomarkers or biomarker panels that are specific to rice intake.

\section{Biomarkers of refined grains}

To our knowledge, there are currently no suggested biomarkers of refined grain intake. This may be due to the fact that most bioactive compounds that could be putative biomarkers are typically located in the outer parts of the grains, and not in the starchy endosperm. The starchy endosperm in cereals contains low amounts of phytochemicals compared with the bran and germ [126]. Moreover, most studies that have been undertaken to find biomarkers of whole grains have used refined grains as a control and it is difficult to use an appropriate control for refined grains per se.

\section{Critical factors that affect biomarker validity and reproducibility - key features of biomarkers}

Biomarker discovery needs to be followed by validation. Several criteria need to be fulfilled before a biomarker can be considered valid, and a framework for the validation of dietary biomarkers have been established [37]. Early validation may include assessment of how specific a biomarker is for a specific food, its pharmacokinetics, dose-response, and its non-dietary determinants. Validity and reproducibility are two features that to a great extent determine the usefulness of a biomarker. Validity is the lack of systematic measurement error when comparing the actual observation with that obtained using a reference method [127]. The correlation between a biomarker measurement and the true intake of the exposure of interest reflects the validity of the biomarker, but since true dietary exposure cannot be estimated without measurement error, the correlation only reflects the upper limit of the validity [128]. However, it should be kept in mind that validity often is study-specific, and therefore it is important to estimate the validity under different conditions in different populations. The reproducibility of a biomarker describes the correlation between samplings within the same individual on independent occasions [38]. The biomarker reproducibility is largely determined by the stability of the individual's intake of the food/nutrient of interest and the elimination half-life of the biomarker. A short half-life can be compensated for by stable and continuous intake [129]. An ideal biomarker should be both valid and reproducible, i.e., plausible and robust and provide an accurate ranking of the intake. A large number of factors affect the accuracy of a biomarker and it is therefore important to evaluate the biomarker before it is used, in order to estimate its reproducibility and validity and identify the factors that affect these parameters. Most food biomarkers fall into the category of concentration biomarkers and the accuracy of such biomarkers are highly variable and dependent on differences in bioavailability of the biomarker substance within and between subjects, differences in metabolism, interactions with other dietary components, differences in distribution volume across subjects as well as the potential impact of gut microbiota on biomarker compounds [130]. It is therefore important to evaluate these aspects of each biomarker before using it as an accurate measure of the intake.

\section{Validation and application of cereal intake biomarkers}

Only very few of the suggested biomarkers of cereal intake have been through rigorous validation. All putative biomarkers covered in this review fall into the category of food intake biomarkers and can be classified as concentration biomarkers [36, 38]. This means that specific intakes are correlated with the concentrations of the biomarkers in the biological matrix investigated and that the biomarkers are affected by inter-personal variation in bioavailability, absorption, metabolism, distribution, and elimination. Moreover, gut microbiota may affect biomarker concentration. Variation inherent to nondietary factors may distort the intake-biomarker relationship and it is important to establish the impact of the different non-dietary determinants as part of the validation process before using the biomarker [60]. For most of the putative biomarkers of cereal intake, this variation remains to be determined. In fact, most of the suggested biomarkers have not been validated at all or only evaluated with regard to some of the criteria [37]. Alkylresorcinols and their metabolites are rare examples of food biomarkers that have been extensively validated as biomarkers of whole-grain wheat and rye intake in various matrixes (alkylresorcinols: plasma, erythrocytes, adipose tissue biopsies; metabolites: plasma and urine) (Table 1). Due to a short-half life (about $5 \mathrm{~h}$ ), they mainly reflect short-term intake, unless the whole-grain wheat and/or rye intake is consumed consistently $(>2$ times per day). Under such conditions, the concentration is stable in fasting plasma samples. Alkylresorcinol concentrations in plasma and adipose tissues as well as their metabolites in plasma and urine samples are well correlated with estimated whole-grain wheat and rye intakes with correlations in the magnitude $0.3-0.55$, depending on the used measure of dietary intake $[31,60,63,64$, 131]. Alkylresorcinols have been found to be a useful complement to traditional dietary assessment methods in several endpoint studies as well as measures as compliance in dietary interventions $[29,31,82,83]$. But it remains to be tested whether they can be combined with other biomarkers to further improve their specificity and 
sensitivity. Moreover, alkylresorcinols in fecal samples have not yet been evaluated as biomarkers. To our knowledge, other biomarker candidates of whole grains, refined grain or fractions of different cereals have not yet been applied as such in endpoint studies. For candidate biomarkers of other grains, more fundamental validation is needed.

\section{Conclusion}

Several biomarker candidates for whole-grain wheat, rye, and oats have been discovered as well as biomarker candidates of fermented rye bread. These biomarker molecules are uniquely found in biological samples from humans after consumption and can be traced down to actual compounds in the food. However, their validity and reliability, which will affect their usefulness as biomarker candidates in epidemiological studies, typically remains to be evaluated under controlled and free-living conditions in humans. Moreover, factors that explain within- and between-person variability in putative biomarkers need to be studied in order to understand their potential and limitations as specific food intake biomarkers. Collection of comprehensive data on lifestyle, health parameters, and OMICs-data including gut microbiota will facilitate the dissection of sources of inter-personal variation and improve the understanding of what factors contribute to inter-individual variation in the ADME of dietary biomarkers.

Metabolomics has enabled simultaneous (semi)quantitation of several biomarkers at the same time in a large number of samples. This allows multi-biomarker signatures to be used as biomarkers rather than single molecules. This approach may have a larger potential to improve specificity and should be further evaluated for different grain intakes. Future studies should evaluate the most suitable matrix (plasma, erythrocytes, urine, hair, nails, or adipose tissue) for determination of specific biomarkers that reflect long-term intake, which is of highest relevance in most diet and health studies.

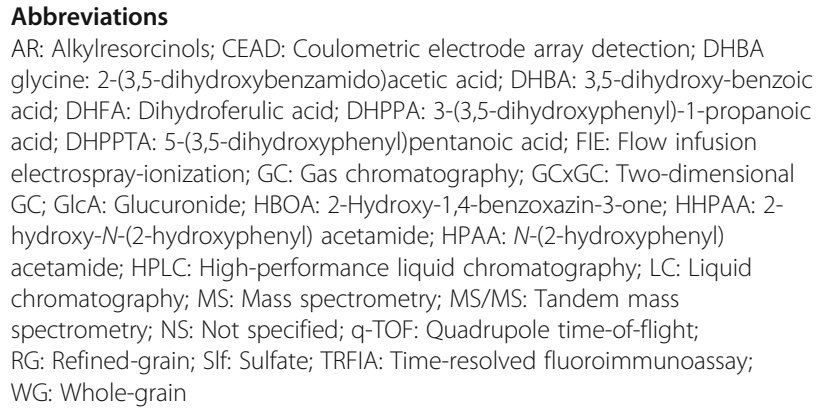
glycine: 2-(3,5-dihydroxybenzamido)acetic acid; DHBA: 3,5-dihydroxy-benzoic acid; DHFA: Dihydroferulic acid; DHPPA: 3-(3,5-dihydroxyphenyl)-1-propanoic acid; DHPPTA: 5-(3,5-dihydroxyphenyl)pentanoic acid; FIE: Flow infusion electrospray-ionization; GC: Gas chromatography; GCxGC: Two-dimensional GC; GlcA: Glucuronide; HBOA: 2-Hydroxy-1,4-benzoxazin-3-one; HHPAA: 2hydroxy-N-(2-hydroxyphenyl) acetamide; HPAA: N-(2-hydroxyphenyl) acetamide; HPLC: High-performance liquid chromatography; LC: Liquid chromatography; MS: Mass spectrometry; MS/MS: Tandem mass spectrometry; NS: Not specified; q-TOF: Quadrupole time-of-flight; RG: Refined-grain; SIf: Sulfate; TRFIA: Time-resolved fluoroimmunoassay; WG: Whole-grain

\section{Acknowledgement}

We would like to acknowledge Professor Lars Dragsted for valuable comments on this manuscript and we acknowledge the JPI-FOODBALL consortium for setting the framework for this and other reviews on dietary biomarkers.

\section{Authors' contributions}

$R L, K H, M G A, L B$, and $M K$ designed the study. $R L, K H, M G A, I B, X Y, L B$, and $M K$ conducted literature search, extracted information, and compiled results in a master Table according to jointly defined criteria. $\mathrm{RL}$ drafted the paper with section contributions from $\mathrm{KH}, \mathrm{MGA}, \mathrm{XY}, \mathrm{IB}$, and $\mathrm{KT}$. All authors contributed with critical intellectual inputs. All authors have read and approved the final manuscript.

\section{Funding}

The work published here was part of the FoodBAll project which is a project funded by the BioNH call (grant number 529051002) under the Joint Programming Initiative "A Healthy Diet for a Healthy Life." The project is funded nationally by the respective research councils; the work was funded in part by a grant from the Spanish National Grants from the Ministry of Economy and Competitiveness (MINECO) (PCIN-2014-133), an award of 2014SGR1566 644 from the Generalitat de Catalunya's Agency AGAUR, and funds from CIBERFES (co-funded by the FEDER program from the EU) to MGA. Project grants from the Swedish Research Council and FORMAS supported the salary of RL. KH was supported by Academy of Finland and Lantmännen Research Foundation. The source of funding has not affected the way data was collected, analyzed, interpreted, or reported.

\section{Availability of data and materials}

This is a review article and does not include original data on humans. Therefore, no original data has been deposited or could be made available. All data have been extracted from published articles referenced in this review.

Ethics approval and consent to participate

This is a review article and does not include original data on humans. Therefore, no ethical approvals have been obtained.

\section{Consent for publication}

This is a review article and does not include original data on humans. Therefore, no consents have been obtained or are required.

\section{Competing interests}

The authors declare that they have no competing interests.

\section{Author details}

'Department of Biology and Biological Engineering, Division of Food and Nutrition Science, Chalmers University of Technology, SE-412 96 Gothenburg, Sweden. ${ }^{2}$ Institute of Public Health and Clinical Nutrition, University of Eastern Finland, P.O. Box 1627, Fl-70211 Kuopio, Finland. ${ }^{3}$ Nutrition and Nutrigenomics Unit, Department of Food Quality and Nutrition, Research and Innovation Centre, Fondazione Edmund Mach, San Michele all'Adige, 38010 Trento, Italy. ${ }^{4}$ Biomarkers and Nutrimetabolomic Laboratory, Department of Nutrition, Food Sciences and Gastronomy, Food Technology Reference Net (XaRTA), Nutrition and Food Safety Research Institute (INSA-UB), Faculty of Pharmacy and Food Sciences, Campus Torribera, University of Barcelona, Barcelona, Spain. ${ }^{5} \mathrm{CIBER}$ de Fragilidad y Envejecimiento Saludable (CIBERFES), Instituto de Salud Carlos III, Barcelona, Spain. ${ }^{6}$ UCD School of Agriculture and Food Science, Institute of Food and Health, Belfield, Dublin 4, Ireland.

Received: 24 February 2019 Accepted: 14 August 2019

\section{References}

1. Lafiandra D, Riccardi G, Shewry PR. Improving cereal grain carbohydrates for diet and health. J Cereal Sci. 2014;59(3):312-26.

2. Van Der Kamp JW, Poutanen K, Seal CJ, Richardson DP. The HEALTHGRAIN definition of "whole grain.". Food Nutr Res. 2014;58(1):22100.

3. Aune D, Chan DS, Lau R, Vieira R, Greenwood DC, Kampman E, et al. Dietary fibre, whole grains, and risk of colorectal cancer: systematic review and dose-response meta-analysis of prospective studies. BMJ. 2011;343:d6617.

4. Aune $D$, Norat $T$, Romundstad $P$, Vatten LJ. Whole grain and refined grain consumption and the risk of type 2 diabetes: a systematic review and dose-response meta-analysis of cohort studies. Eur J Epidemiol. 2013;28(11):845-58

5. Benisi-Kohansal S, Saneei P, Salehi-Marzijarani M, Larijani B, Esmaillzadeh A Whole-grain intake and mortality from all causes, cardiovascular disease, 
and cancer: a systematic review and dose-response meta-analysis of prospective cohort studies. Adv Nutr. 2016;7(6):1052-65.

6. Pol K, Christensen R, Bartels EM, Raben A, Tetens I, Kristensen M. Whole grain and body weight changes in apparently healthy adults: a systematic review and meta-analysis of randomized controlled the European Union Sixth Framework Program Integrated Project. Amrican J Clin Nutr. 2013: 98(4):872-84.

7. Reynolds A, Mann J, Cummings J, Winter N, Mete E, Te Morenga L. Carbohydrate quality and human health: a series of systematic reviews and meta-analyses. Lancet. 2019;393(10170):434-45.

8. Chen J, Huang Q, Shi W, Yang L, Chen J, Lan Q. Meta-analysis of the association between whole and refined grain consumption and stroke risk based on prospective cohort studies. Asia-Pacific J Public Heal. 2016;28(7):563-75.

9. Hu EA, Pan A, Malik V, Sun Q. White rice consumption and risk of type 2 diabetes: meta-analysis and systematic review. BMJ. 2012;344(7851):1-9.

10. Okarter N, Liu RH. Health benefits of whole grain phytochemicals. Crit Rev Food Sci Nutr. 2010;50(3):193-208.

11. Johansson DP, Gutiérrez JLV, Landberg R, Alminger M, Langton M. Impact of food processing on rye product properties and their in vitro digestion. Eur J Nutr. 2018;57(4):1651-66.

12. Bindels LB, Walter J, Ramer-Tait AE. Resistant starches for the management of metabolic diseases. Curr Opin Clin Nutr Metab Care. 2015;18(6):559-65.

13. Sandberg JC, Björck IME, Nilsson AC. Effects of whole grain rye, with and without resistant starch type 2 supplementation, on glucose tolerance, gut hormones, inflammation and appetite regulation in an 11-14.5 hour perspective; a randomized controlled study in healthy subjects. Nutr J. 2017; 16(1):1-11.

14. Aune D, Keum N, Giovannucci E, Fadnes LT, Boffetta P, Greenwood DC, et al. Whole grain consumption and risk of cardiovascular disease, cancer, and all cause and cause specific mortality: systematic review and doseresponse meta-analysis of prospective studies. BMJ. 2016;353:12716.

15. Helnæs A, Kyrø C, Andersen I, Lacoppidan S, Overvad K, Christensen J, et al. Intake of whole grains is associated with lower risk of myocardial infarction: The Danish Diet, Cancer and Health Cohort. Am J Clin Nutr. 2016;103(4): 999-1007.

16. Kelly S, Hartley L, Emma L, Jill LC, Helen MJ, Al-Khudairy L, et al. Whole grain cereals for the primary or secondary prevention of cardiovascular disease. Cochrane Database Syst Rev. 2017:8:CD005051.

17. Marventano S, Vetrani C, Vitale M, Godos J, Riccardi G, Grosso G. Whole grain intake and glycaemic control in healthy subjects: a systematic review and meta-analysis of randomized controlled trials. Nutrients. 2017;9:769.

18. Kristensen M, Pelletier $X$, Ross AB, Thielecke F. A high rate of noncompliance confounds the study of whole grains and weight maintenance in a randomised intervention trial-the case for greater use of dietary biomarkers in nutrition intervention studies. Nutrients. 2017:9:55.

19. Marklund M, Magnusdottir OK, Rosqvist F, Cloetens L, Landberg R, Kolehmainen $\mathrm{M}$, et al. A dietary biomarker approach captures compliance and cardiometabolic effects of a healthy Nordic diet in individuals with metabolic syndrome. J Nutr. 2014;144(10):1642-9.

20. Kyrø C, Skeie G, Dragsted LO, Christensen J, Overvad K, Hallmans G, et al. Intake of whole grains in Scandinavia is associated with healthy lifestyle, socio-economic and dietary factors. Public Health Nutr. 2011;14(10):1787-95.

21. Andersson A, Tengblad S, Karlstro B, Kamal-eldin A, Landberg R, Basu S, et al. Whole-grain foods do not affect insulin sensitivity or markers of lipid peroxidation and Inflammation in healthy, moderately overweight subjects. J Nutr. 2007;137:1401-7.

22. Brownlee IA, Moore C, Chatfield M, Richardson DP, Ashby P, Kuznesof SA, et al. Markers of cardiovascular risk are not changed by increased wholegrain intake: the WHOLEheart study, a randomised, controlled dietary intervention. Br J Nutr. 2010;104(1):125-34.

23. Tighe P, Duthie G, Vaughan N, Brittenden J, Simpson WG, Duthie S, et al Effect of increased consumption of whole-grain foods on blood pressure and other cardiovascular risk markers in healthy middle-aged persons: a randomized controlled trial. Am J Clin Nutr. 2010;92(4):733-40.

24. Len M, Slavin JL, Fulcher RG, editors. Dietary intake of whole grains: a challenge for consumers, Whole-grain foods in health and disease: American Association of Cereal Chemists; 2002. p. 301-25.

25. Frølich W, Åman P. Whole grain for whom and why? Food Nutr Res. 2010; 54:1-5.

26. Lang R, Jebb SA. Who consumes whole grains, and how much? Proc Nutr Soc. 2003;62(01):123-7.
27. Ross AB, van der Kamp J-W, King R, Lê K-A, Mejborn H, Seal CJ, et al. Perspective: a definition for whole-grain food products-recommendations from the Healthgrain Forum. Adv Nutr. 2017:8(4):525-31.

28. Jensen MK, Koh-Banerjee P, Hu FB, Franz M, Sampson L, Grønbæk M. Intakes of whole grains, bran, and germ and the risk of coronary heart disease in men. Am J Clin Nutr. 2004;7:1492-9.

29. Biskup I, Kyrø C, Marklund M, Olsen A, Van Dam RM, Tjønneland A, et al. Plasma alkylresorcinols, biomarkers of whole-grain wheat and rye intake, and risk of type 2 diabetes in Scandinavian men and women. Am J Clin Nutr. 2016;104(1):88-96.

30. Landberg R, Kamal-Eldin A, Andersson A, Vessby B, Aman P. Alkylresorcinols as biomarkers of whole-grain wheat and rye intake: plasma concentration and intake estimated from dietary records. Am J Clin Nutr. 2008;87(4):832-8.

31. Ross AB, Bourgeois A, Macharia HN, Kochhar S, Jebb SA, Brownlee IA, et al. Plasma alkylresorcinols as a biomarker of whole-grain food consumption in a large population: results from the WHOLEheart Intervention Study. Am J Clin Nutr. 2012;95(1):204-11.

32. Magnusdottir OK, Landberg R, Gunnarsdottir I, Cloetens L, Akesson B, Rosqvist $F$, et al. Whole grain rye intake, reflected by a biomarker, is associated with favorable blood lipid outcomes in subjects with the metabolic syndrome - a randomized study. PLoS One. 2014;9(10):e110827.

33. Freedman LS, Kipnis V, Schatzkin A, Tasevska N. Can we use biomarkers in combination with self-reports to strengthen the analysis of nutritional epidemiological studies? Epidemiol Perspect Innov. 2010;7:2.

34. Sang S. Biomarkers of whole grain intake. J Agric Food Chem. 2018;66(40): 10347-52.

35. Moher D, Liberati A, Tetzlaff J, Altman DG, Altman D, Antes G, et al. Preferred reporting items for systematic reviews and meta-analyses: The PRISMA statement. PLoS Med. 2009;6(7):e1000097.

36. Gao Q, Praticò $G$, Scalbert A, Vergères $G$, Kolehmainen M, Manach C, et al. A scheme for a flexible classification of dietary and health biomarkers. Genes Nutr. 2017;12(1):1-15.

37. Dragsted LO, Gao Q, Scalbert A, Vergères G, Kolehmainen M, Manach C, et al. Validation of biomarkers of food intake-Critical assessment of candidate biomarkers. Genes Nutr. 2018;13(1):14.

38. Kaaks R, Riboli E, Sinha R. Biochemical markers of dietary intake. IARC Sci Publ. 1997;142:103-26.

39. Neuhouser ML, Tinker L, Shaw PA, Schoeller D, Bingham SA, Van Horn L, et al. Use of recovery biomarkers to calibrate nutrient consumption selfreports in the Women's Health Initiative. Am J Epidemiol. 2008;167(10): 1247-59.

40. Tasevska N. Urinary sugars - a biomarker of total sugars intake. Nutrients. 2015;7(7):5816-33.

41. Jenab M, Slimani N, Bictash M, Ferrari P, Bingham SA. Biomarkers in nutritional epidemiology: applications, needs and new horizons. Hum Genet. 2009;125(5-6):507-25.

42. Guasch-Ferre M, Bhupathiraju SN, Hu FB. Use of metabolomics in improving assessment of dietary intake. Clin Chem. 2018;64(1):82-98.

43. Scalbert A, Brennan L, Manach C, Andres-Lacueva C, Dragsted LO, Draper J, et al. The food metabolome: a window over dietary exposure. Am J Clin Nutr. 2014;99:1286-308.

44. Brunius C, Wu H, Landberg R. Targeted and untargeted metabolomics for specific food intake assessment. In: Schoeller DA, Westerterp M, editors Advances in the Assessment of Dietary Intake. USA: CRC Press, Taylor \& Francis; 2017. p. 315-36.

45. Patti GJ, Yanes O, Siuzdak G. Metabolomics: the apogee of the omics trilogy. Nat Rev Mol Cell Biol. 2012;13:263-9.

46. Landberg R, Marklund M, Kamal-Eldin A, Åman P. An update on alkylresorcinols - occurrence, bioavailability, bioactivity and utility as biomarkers. J Funct Foods. 2014;7(0):77-89.

47. Ross AB, Svelander C, Karlsson G, Savolainen OI. Identification and quantification of even and odd chained 5-n alkylresorcinols, branched chain-alkylresorcinols and methylalkylresorcinols in Quinoa (Chenopodium quinoa). Food Chem. 2017;220:344-51.

48. Wang P, Yang J, Yerke A, Sang S. Avenacosides: Metabolism, and potential use as exposure biomarkers of oat intake. Mol Nutr Food Res. 2017:61(7):1-10.

49. Sang $S$, Wang $P$, Yerke A. Avenanthramides and their microbial metabolites as the urinary exposure markers for whole grain oat intake: a kinetic study in humans. FASEB J. 2017;31(1_supplement):646.19.

50. Bondia-Pons I, Barri T, Hanhineva K, Juntunen K, Dragsted LO, Mykkänen H, et al. UPLC-QTOF/MS metabolic profiling unveils urinary changes in humans 
after a whole grain rye versus refined wheat bread intervention. Mol Nutr Food Res. 2013;57(3):412-22.

51. Hanhineva K, Brunius C, Andersson A, Marklund M, Juvonen R, KeskiRahkonen $\mathrm{P}$, et al. Discovery of urinary biomarkers of whole grain rye intake in free-living subjects using nontargeted LC-MS metabolite profiling. Mol Nutr Food Res. 2015; n/a-n/a.

52. Hanhineva K, Keski-Rahkonen P, Lappi J, Katina K, Pekkinen J, Savolainen O, et al. The postprandial plasma rye fingerprint includes benzoxazinoidderived phenylacetamide sulfates. J Nutr. 2014;144(7):1016-22.

53. Hanhineva $K$, Lankinen MA, Pedret A, Schwab U, Kolehmainen M, Paananen J, et al. Nontargeted metabolite profiling discriminates diet-specific biomarkers for consumption of whole grains, fatty fish, and bilberries in a randomized controlled trial. J Nutr. 2015;145:7-17.

54. Zhu $Y$, Wang $P$, Sha $W$, Sang S. Urinary biomarkers of whole grain wheat intake identified by non-targeted and targeted metabolomics approaches. Sci Rep. 2016;6(November):1-16.

55. Ross AB, Pineau N, Kochhar S, Bourgeois A, Beaumont M, Decarli B. Validation of a FFQ for estimating whole-grain cereal food intake. Br J Nutr. 2009;102(11):1547-51.

56. Jackson KH, West SG, Vanden Heuvel JP, Jonnalagadda SS, Ross AB, Hill AM, et al. Effects of whole and refined grains in a weight-loss diet on markers of metabolic syndrome in individuals with increased waist circumference: a randomized controlled-feeding trial. Am J Clin Nutr. 2014;100(2):577-86.

57. Ampatzoglou A, Atwal KK, Maidens CM, Williams CL, Ross AB, Thielecke F, et al. Increased whole grain consumption does not affect blood biochemistry, body composition, or gut microbiology in healthy, lowhabitual whole grain consumers 1 - 3. J Nutr. 2015;145:215-21.

58. Knudsen MD, Kyrø C, Olsen A, Dragsted LO, Skeie G, Lund E, et al. Selfreported whole-grain intake and plasma alkylresorcinol concentrations in combination in relation to the incidence of colorectal cancer. Am J Epidemiol. 2014;179(10):1188-96.

59. Ross AB, Bruce SJ, Blondel-Lubrano A, Oguey-Araymon S, Beaumont M, Bourgeois A, et al. A whole-grain cereal-rich diet increases plasma betaine, and tends to decrease total and LDL-cholesterol compared with a refinedgrain diet in healthy subjects. Br J Nutr. 2011;105:1492-502.

60. Landberg R, Kamal-Eldin A, Christensen J, Overvad KIM, Tj A, Olsen A, et al. Determinants of plasma alkylresorcinol concentration in Danish postmenopausal women. Eur J Clin Nutr. 2011;65:94-101.

61. Ma J, Ross AB, Shea MK, Bruce SJ, Jacques PF, Saltzman E, et al. Plasma alkylresorcinols, biomarkers of whole-grain intake, are related to lower BMI in older adults. J Nutr. 2012;142(10):1859-64.

62. Jansson E, Landberg R, Kamal-Eldin A, Wolk A, Vessby B, Aman P. Presence of alkylresorcinols, potential whole grain biomarkers, in human adipose tissue. Br J Nutr. 2010;104(5):633-6.

63. Landberg R, Townsend MK, Neelakantan N, Sun Q, Sampson L, Spiegelman $D$, et al. Alkylresorcinol metabolite concentrations in spot urine samples correlated with whole grain and cereal fiber intake but showed low to modest reproducibility over one to three years in U.S. women. J Nutr. 2012; 142:872-7.

64. Marklund M, Landberg R, Andersson A, Åman P, Kamal-Eldin A. Alkylresorcinol metabolites in urine correlate with the intake of whole grains and cereal fibre in free-living Swedish adults. Br J Nutr. 2013;109(1): 129-36.

65. Drake I, Sonestedt E, Gullberg B, Bjartell A, Olsson H, Adlercreutz H, et al. Plasma alkylresorcinol metabolites as biomarkers for whole-grain intake and their association with prostate cancer: a Swedish nested case-control study. Cancer Epidemiol Biomarkers Prev. 2014;23(1):73-83.

66. Guyman LA, Adlercreutz H, Koskela A, Li L, SAA B, Lampe JW. Urinary 3-(3,5dihydroxyphenyl)-1-propanoic acid, an alkylresorcinol metabolite, is a potential biomarker of whole-grain intake in a U.S. population. J Nutr. 2018; 138(10):1957-62.

67. Garcia-Aloy M, Llorach R, Urpi-Sarda M, Tulipani S, Salas-Salvadó J, MartínezGonzález MA, et al. Nutrimetabolomics fingerprinting to identify biomarkers of bread exposure in a free-living population from the PREDIMED study cohort. Metabolomics. 2014;11(1):155-65.

68. Altorf-van der Kuil W, Brink EJ, Boetje M, Siebelink E, Bijlsma S, Engberink $M F$, et al. Identification of biomarkers for intake of protein from meat, dairy products and grains: a controlled dietary intervention study. Br J Nutr. 2013; 110(5):810-22

69. Linko A-M, Adlercreutz $\mathrm{H}$. Whole-grain rye and wheat alkylresorcinols are incorporated into human erythrocyte membranes. Br J Nutr. 2005;93(01):11.
70. Andersson A, Marklund M, Diana M, Landberg R. Plasma alkylresorcinol concentrations correlate with whole grain wheat and rye intake and show moderate reproducibility over a 2 - to 3 -month period in free-living Swedish adults. J Nutr. 2011;141:1712-8.

71. Linko A-M, Juntunen KS, Mykkanen HM, Adlercreutz H. Whole-grain rye bread consumption by women correlates with plasma alkylresorcinols and increases their concentration compared with low-fiber wheat bread. J Nutr. 2005;135(3):580-3.

72. Kristensen M, Toubro S, Jensen MG, Ross AB, Riboldi G, Petronio M, et al. Whole grain compared with refined wheat decreases the percentage of body fat following a 12-week, energy-restricted dietary intervention in postmenopausal women. J Nutr. 2012;142(4):710-6.

73. Meija L, Krams I, Cauce V, Samaletdin A, Söderholm P, Meija R, et al. Alkylresorcinol metabolites in urine and plasma as potential biomarkers of rye and wheat fiber consumption in prostate cancer patients and controls. Nutr Cancer. 2015;67(2):258-65.

74. Söderholm PP, Alfthan G, Tikkanen MJ, Adlercreutz H. Rye bread intake improves oxidation resistance of $L D L$ in healthy humans. Atherosclerosis. 2012;221(2):583-6.

75. Söderholm PP, Lundin JE, Koskela AH, Tikkanen MJ, Adlercreutz HC. Pharmacokinetics of alkylresorcinol metabolites in human urine. $\mathrm{Br} J$ Nutr. 2011;106(7):1040-4.

76. Söderholm PP, Koskela AH, Lundin JE, Tikkanen MJ, Adlercreutz HC. Plasma pharmacokinetics of alkylresorcinol metabolites: new candidate biomarkers for whole-grain rye and wheat intake. Am J Clin Nutr. 2009;90(5):1167-71.

77. Aubertin-Leheudre M, Koskela A, Samaletdin A, Adlercreutz $H$ Responsiveness of urinary and plasma alkylresorcinol metabolites to rye intake in finnish women. Cancers (Basel). 2010;2(2):513-22.

78. Marklund M, Landberg R, Andersson R, Aman P, Kamal-Eldin A. Alkylresorcinol metabolism in Swedish adults is affected by factors other than intake of whole-grain wheat and rye. J Nutr. 2012;142(8):1479-86.

79. Zhu Y, Shurlknight KL, Chen X, Sang S. Identification and pharmacokinetics of novel alkylresorcinol metabolites in human urine, new candidate biomarkers for whole-grain wheat and rye intake. J Nutr. 2014;144(9):114-22.

80. Beckmann M, Lloyd AJ, Haldar S, Seal C, Brandt K, Draper J. Hydroxylated phenylacetamides derived from bioactive benzoxazinoids are bioavailable in humans after habitual consumption of whole grain sourdough rye bread. Mol Nutr Food Res. 2013;57(10):1859-73.

81. Vitaglione P, Mennella I, Ferracane R, Rivellese AA, Giacco R, Ercolini D, et al. Whole-grain wheat consumption reduces inflammation in a randomized controlled trial on overweight and obese subjects with unhealthy dietary and lifestyle behaviors: role of polyphenols bound to cereal dietary fibe. Am J Clin Nutr. 2015;101:251-61.

82. McKeown NM, Hruby A, Landberg R, Herrington DM, Lichtenstein AH. Plasma alkylresorcinols, biomarkers of whole-grain intake, are not associated with progression of coronary artery atherosclerosis in postmenopausal women with coronary artery disease. Public Health Nutr. 2015:1-6.

83. Kyrø C, Olsen A, Landberg R, Skeie G, Loft S, Åman P, et al. Plasma alkylresorcinols, biomarkers of whole-grain wheat and rye intake, and incidence of colorectal cancer. J Natl Cancer Inst. 2014;106(1):1-9.

84. Kyrø C, Olsen A, Bueno-de-Mesquita HBA, Skeie G, Loft S, Åman P, et al. Plasma alkylresorcinol concentrations, biomarkers of whole-grain wheat and rye intake, in the European Prospective Investigation into Cancer and Nutrition (EPIC) cohort. Br J Nutr. 2014;111(10):1881-90.

85. Landberg R, Åman P, Friberg LE, Vessby B, Adlercreutz H, Kamal-Eldin A. Dose response of whole-grain biomarkers: alkylresorcinols in human plasma and their metabolites in urine in relation to intake. Am J Clin Nutr. 2009; 89(5):1478.

86. Landberg R, Linko A-M, Kamal-Eldin A, Vessby B, Adlercreutz H, Aman P. Human plasma kinetics and relative bioavailability of alkylresorcinols after intake of rye bran. J Nutr. 2006;136(11):2760-5.

87. Aubertin-Leheudre M, Koskela A, Marjamaa A, Adlercreutz H. Plasma alkylresorcinols and urinary alkylresorcinol metabolites as biomarkers of cereal fiber intake in finnish women. Cancer Epidemiol Biomarkers Prev. 2008;17(9):2244-8

88. Aubertin-Leheudre M, Koskela A, Samaletdin A, Adlercreutz H. Plasma alkylresorcinol metabolites as potential biomarkers of whole-grain wheat and rye cereal fibre intakes in women. Br J Nutr. 2010;103:339-43.

89. Johansson-Persson A, Barri T, Ulmius M, Önning G, Dragsted LO. LC-QTOF/ MS metabolomic profiles in human plasma after a 5-week high dietary fiber intake. Anal Bioanal Chem. 2013;405(14):4799-809. 
90. Harder H, Tetens I, Let MB, Meyer AS. Rye bran bread intake elevates urinary excretion of ferulic acid in humans, but does not affect the susceptibility of LDL to oxidation ex vivo. Eur J Nutr. 2004;43(4):230-6.

91. Anson NM, Selinheimo E, Havenaar R, Aura AM, Mattila I, Lehtinen P, et al. Bioprocessing of wheat bran improves in vitro bioaccessibility and colonic metabolism of phenolic compounds. J Agric Food Chem. 2009:57(14):6148-55.

92. Lappi J, Aura AM, Katina K, Nordlund E, Kolehmainen M, Mykkänen H, et al. Comparison of postprandial phenolic acid excretions and glucose responses after ingestion of breads with bioprocessed or native rye bran. Food Funct. 2013:4(6):972-81.

93. Sheflin AM, Borresen EC, Wdowik MJ, Rao S, Brown RJ, Heuberger AL, et al. Pilot dietary intervention with heat-stabilized rice bran modulates stool microbiota and metabolites in healthy adults. Nutrients. 2015;7(2):1282-300.

94. Ross A, Savolainen O. A new biomarker for quinoa intake. In: The FASEB Journal; 2014

95. Sonestedt E, Borgquist S, Ericson U, Gullberg B, Olsson H, Adlercreutz H, et al. Enterolactone is differently associated with estrogen receptor $\beta$ negative and -positive breast cancer in a Swedish nested case-control study. Cancer Epidemiol Biomarkers Prev. 2008;17(11):3241-51.

96. Mclntosh GH, Noakes M, Royle PJ, Foster PR. Whole-grain rye and wheat foods and markers of bowel health in overweight middle-aged men. Am J Clin Nutr. 2003;77(4):967-74.

97. Durazzo A, Carcea M, Adlercreutz H, Azzini E, Polito A, Olivieri L, et al. Effects of consumption of whole grain foods rich in lignans in healthy postmenopausal women with moderate serum cholesterol: a pilot study. Int J Food Sci Nutr. 2014;65(5):637-45.

98. De Carvalho FG, Ovídio PP, Padovan GJ, Jordão Junior AA, Marchini JS, Navarro AM. Metabolic parameters of postmenopausal women after quinoa or corn flakes intake-a prospective and double-blind study. Int J Food Sci Nutr. 2014;65(3):380-5.

99. Cassani RSL, Fassini PG, Silvah JH, Lima CMM, Marchini JS. Impact of weight loss diet associated with flaxseed on inflammatory markers in men with cardiovascular risk factors: A clinical study. Nutr J. 2015;14(1):1-8.

100. Landberg R, Kamal-Eldin A, Salmenkallio-Marttila M, Rouau X, Åman P. Localization of alkylresorcinols in wheat, rye and barley kernels. J Cereal Sci. 2008;48(2):401-6.

101. Wierzbicka R, Wu H, Franek M, Kamal-Eldin A, Landberg R. Determination of alkylresorcinols and their metabolites in biological samples by gas chromatography-mass spectrometry. J Chromatogr B. 2015;1000:120-9.

102. Wierzbicka R, Zamaratskaia G, Kamal-Eldin A, Landberg R. Novel urinary alkylresorcinol metabolites as biomarkers of whole grain intake in free-living Swedish adults. Mol Nutr Food Res. 2017;61(7):1-8.

103. Chen $Y$, Ross AB, Aman P, Kamal-Eldin A. Alkylresorcinols as markers of whole grain wheat and rye in cereal products. J Agric Food Chem. 2004; 52(26):8242-6.

104. Landberg R, Kamal-Eldin A, Andersson R, Åman P. Alkylresorcinol content and homologue composition in durum wheat (Triticum durum) kernels and pasta products. J Agric Food Chem. 2006;54(8):3012-4.

105. Landberg R, Aman P, Hallmans G, Johansson I. Long-term reproducibility of plasma alkylresorcinols as biomarkers of whole-grain wheat and rye intake within Northern Sweden Health and Disease Study Cohort. Eur J Clin Nutr. 2013;67(3):259-63.

106. Marklund M, Stromberg EA, Lærke HN, KEB K, Kamal-eldin A. Simultaneous pharmacokinetic modeling of alkylresorcinols and their main metabolites indicates dual absorption mechanisms and enterohepatic elimination in humans. J Nutr. 2014;144(12):1674-80.

107. Sun Q, Bertrand KA, Franke AA, Rosner B, Curhan GC, Willett WC. Reproducibility of urinary biomarkers in multiple 24-h urine samples. Am J Clin Nutr. 2017;105(1):159-68.

108. Adhikari KB, Tanwir F, Gregersen PL, Steffensen SK, Jensen BM, Poulsen LK, et al. Benzoxazinoids: cereal phytochemicals with putative therapeutic and health-protecting properties. Mol Nutr Food Res. 2015; n/a-n/a.

109. Dihm K, Vendelbo Lind M, Sundén H, Ross A, Savolainen O. Quantification of benzoxazinoids and their metabolites in Nordic breads. Food Chem. 2017;235:7-13

110. Savolainen O, Pekkinen J, Katina K, Poutanen K, Hanhineva K. Glycosylated benzoxazinoids are degraded during fermentation of wheat bran. J Agric Food Chem. 2015;63(25):5943-9.

111. Steffensen SK, Pedersen HA, Adhikari KB, Laursen BB, Jensen C, Høyer S, et al. Benzoxazinoids in prostate cancer patients after a rye-intensive diet: methods and initial results. J Agric Food Chem. 2016;64(43):8235-45.
112. Cortés-Martín A, García-Villalba R, González-Sarrías A, Romo-Vaquero M, Loria-Kohen V, Ramírez-De-Molina A, et al. The gut microbiota urolithin metabotypes revisited: the human metabolism of ellagic acid is mainly determined by aging. Food Funct. 2018;9(8):4100-6.

113. Sang S, Chu YF. Whole grain oats, more than just a fiber: role of unique phytochemicals. Mol Nutr Food Res. 2017;61(7):1-12.

114. Chen C-YO, Milbury PE, Collins FW, Blumberg JB. Avenanthramides are bioavailable and have antioxidant activity in humans after acute consumption of an enriched mixture from oats. J Nutr. 2007;137(6):1375-82.

115. Zhang T, Shao J, Gao Y, Chen C, Yao D, Chu YF, et al. Absorption and elimination of oat avenanthramides in humans after acute consumption of oat cookies. Oxid Med Cell Longev. 2017;2017:2056705.

116. Walsh J, Haddock J, Blumberg JB, McKay DL, Wei X, Dolnikowski G, et al. Identification of methylated metabolites of oat avenanthramides in human plasma using UHPLC QToF-MS. Int J Food Sci Nutr. 2018;69(3):377-83.

117. Schär MY, Corona G, Soycan G, Dine C, Kristek A, Alsharif SNS, et al. Excretion of avenanthramides, phenolic acids and their major metabolites following intake of oat bran. Mol Nutr Food Res. 2018;62(2):1-9.

118. Yang J, Wang P, Wu W, Zhao Y, Idehen E, Sang S. Steroidal saponins in oat bran. J Agric Food Chem. 2016;64(7):1549-56.

119. López DN, Galante M, Robson M, Boeris V, Spelzini D. Amaranth, quinoa and chia protein isolates: physicochemical and structural properties. Int J Biol Macromol. 2018;109:152-9.

120. Tang $Y$, Tsao R. Phytochemicals in quinoa and amaranth grains and their antioxidant, anti-inflammatory, and potential health beneficial effects: a review. Mol Nutr Food Res. 2017;61(7):1-16

121. Andersson A, Lampi A, Nystro L, Piironen V, Li L, Ward JL, et al. Phytochemical and dietary fiber components in barley varieties in the HEALTHGRAIN diversity screen. J Agric Food Chem. 2008;56:9767-76.

122. Lind MV, Madsen ML, Rumessen JJ, Vestergaard H, Gobel RJ, Hansen T, et al. Plasma alkylresorcinols reflect gluten intake and distinguish between gluten-rich and gluten-poor diets in a population at risk of metabolic syndrome. J Nutr. 2016;146(10):1991-8.

123. Guertin KA, Moore SC, Sampson JN, Huang WY, Xiao Q, StolzenbergSolomon RZ, et al. Metabolomics in nutritional epidemiology: identifying metabolites associated with diet and quantifying their potential to uncover diet-disease relations in populations. Am J Clin Nutr. 2014;100(1):208-17.

124. Li KJ, Borresen EC, Jenkins-Puccetti N, Luckasen G, Ryan EP. Navy bean and rice bran intake alters the plasma metabolome of children at risk for cardiovascular disease. Front Nutr. 2018;4:71

125. Brown DG, Borresen EC, Brown RJ, Ryan EP. Heat-stabilised rice bran consumption by colorectal cancer survivors modulates stool metabolite profiles and metabolic networks: a randomised controlled trial. Br J Nutr. 2017:117(9):1244-56.

126. Coulomb M, Gombert A, Moazzami AA. Metabolomics study of cereal grains reveals the discriminative metabolic markers associated with anatomical compartments. Ital J Food Sci. 2015;27(2):142-50.

127. Vineis $P, G$ Gallo V. Epidemiological concepts of validation of biomarkers for the identification/quantification of environmental carcinogenic exposures. Poland: ECNIS; 2007.

128. Kaaks R, Ferrari P, Ciampi A, Plummer M, Riboli E. Uses and limitations of statistical accounting for random error correlations, in the validation of dietary questionnaire assessments. Public Health Nutr. 2002;5(6a):969-76.

129. van Dam R, Hu FB. Are alkylresorcinols accurate biomarkers for whole grain intake? Am J Clin Nutr. 2008:87:797-8.

130. Brouwer-Brolsma EM, Brennan L, Drevon CA, Van Kranen H, Manach C, Dragsted LO, et al. Combining traditional dietary assessment methods with novel metabolomics techniques: present efforts by the Food Biomarker Alliance. Proc Nutr Soc. 2017:76(4):619-27.

131. Your microbes at work: fiber fermenters keep us healthy. Nature. 2015;518:S9.

\section{Publisher's Note}

Springer Nature remains neutral with regard to jurisdictional claims in published maps and institutional affiliations. 